\title{
Added Value of Electronic Immunization Registries in Low- and Middle-Income Countries: Observational Case Study in Tanzania
}

Andrew M Secor ${ }^{1}$, MPH; Hassan Mtenga ${ }^{2}$, MPH; John Richard ${ }^{2}$, BSc; Ngwegwe Bulula ${ }^{3}$, MSc; Ellen Ferriss ${ }^{1}$, MS; Mansi Rathod ${ }^{1}$, MS; Tove K Ryman ${ }^{4}$, MPH, PhD; Laurie Werner ${ }^{1}$, MPA; Emily Carnahan ${ }^{1}$, MPH

${ }^{1}$ PATH, Seattle, WA, United States

${ }^{2}$ PATH, Dar es Salaam, United Republic of Tanzania

${ }^{3}$ Immunization and Vaccine Development Program, Ministry of Health, Community Development, Gender, Elderly and Children, Dar es Salaam, United Republic of Tanzania

${ }^{4}$ Bill \& Melinda Gates Foundation, Seattle, WA, United States

Corresponding Author:

Emily Carnahan, MPH

PATH

2201 Westlake Avenue

Seattle, WA,

United States

Phone: 12062853500

Email: ecarnahan@path.org

\section{Abstract}

Background: There is growing interest and investment in electronic immunization registries (EIRs) in low- and middle-income countries. EIRs provide ready access to patient- and aggregate-level service delivery data that can be used to improve patient care, identify spatiotemporal trends in vaccination coverage and dropout, inform resource allocation and program operations, and target quality improvement measures. The Government of Tanzania introduced the Tanzania Immunization Registry (TImR) in 2017, and the system has since been rolled out in 3736 facilities in 15 regions.

Objective: The aims of this study are to conceptualize the additional ways in which EIRs can add value to immunization programs (beyond measuring vaccine coverage) and assess the potential value-add using EIR data from Tanzania as a case study.

Methods: This study comprised 2 sequential phases. First, a comprehensive list of ways EIRs can potentially add value to immunization programs was developed through stakeholder interviews. Second, the added value was evaluated using descriptive and regression analyses of TImR data for a prioritized subset of program needs.

Results: The analysis areas prioritized through stakeholder interviews were population movement, missed opportunities for vaccination (MOVs), continuum of care, and continuous quality improvement. The included TImR data comprised 958,870 visits for 559,542 patients from 2359 health facilities. Our analyses revealed that few patients sought care outside their assigned facility $(44,733 / 810,568,5.52 \%$ of applicable visits); however, this varied by region; facility urbanicity, type, ownership, patient volume, and duration of TImR system use; density of facilities in the immediate area; and patient age. Analyses further showed that MOVs were highest among children aged < 12 months $(215,576 / 831,018,25.94 \%$ of visits included an MOV and were applicable visits); however, there were few significant differences based on other individual or facility characteristics. Nearly half $(133,337 / 294,464$, $45.28 \%$ ) of the children aged 12 to 35 months were fully vaccinated or had received all doses except measles-containing vaccine-1 of the 14-dose under-12-month schedule (ie, through measles-containing vaccine-1), and facility and patient characteristics associated with dropout varied by vaccine. The continuous quality improvement analysis showed that most quality issues (eg, MOVs) were concentrated in $<10 \%$ of facilities, indicating the potential for EIRs to target quality improvement efforts.

Conclusions: EIRs have the potential to add value to immunization stakeholders at all levels of the health system. Individual-level electronic data can enable new analyses to understand service delivery or care-seeking patterns, potential risk factors for underimmunization, and where challenges occur. However, to achieve this potential, country programs need to leverage and strengthen the capacity to collect, analyze, interpret, and act on the data. As EIRs are introduced and scaled in low- and middle-income countries, implementers and researchers should continue to share real-world examples and build an evidence base for how EIRs can add value to immunization programs, particularly for innovative uses. 
(JMIR Public Health Surveill 2022;8(1):e32455) doi: 10.2196/32455

\section{KEYWORDS}

immunization; immunization information system; electronic immunization registry; digital health; eHealth

\section{Introduction}

\section{Background}

With the increasing digitalization of health systems worldwide, there is growing interest and investment in electronic immunization registries (EIRs). EIRs are "confidential, computerized, population-based systems that collect and consolidate vaccination data from vaccination providers for better immunization strategies" [1]. EIRs are designed to provide a consolidated patient record to health care workers at the point of care to enable the delivery of the appropriate vaccines at the appropriate time. At the population level, EIRs can provide aggregate data on vaccination coverage to inform resource allocation and program operations. In this way, EIRs aim to improve the immunization delivery system to reach every child by supporting more effective, efficient, and data-driven care $[2,3]$.

Vaccine coverage has historically been the primary metric for evaluating immunization programs. As an increasing number of low- and middle-income countries (LMICs) have begun implementing EIRs, vaccine coverage has been measured as a key outcome for assessing EIR effectiveness. Pre-post studies in Vietnam, Bangladesh, and Pakistan have demonstrated significant increases in child vaccination coverage after the introduction of EIRs that included SMS text message reminders and, in addition, in Pakistan, decision support systems [4-6].

In addition to improving vaccine coverage, other benefits of EIRs have been identified for individuals, immunization program performance and management, research, and population health $[7,8]$. For example, EIRs store patient vaccine history at the individual level, can help identify defaulters and reduce dropout rates at the program level, and provide data to support resource allocation and strategic planning at the population level [8]. Across levels, EIRs that capture individual-level data provide an opportunity to redefine traditional vaccine indicators and conduct more timely, granular analyses to support decision-making [9]. EIRs enable immunization programs to explore outcomes of interest beyond vaccine coverage, including longitudinal outcomes at the population and individual levels. As EIRs are costly to introduce and maintain, it is important for decision-makers to consider all possible benefits to justify the investment $[10,11]$.

In some settings where EIRs are being considered or introduced, immunization coverage may already be high and, therefore, not an appropriate metric for EIR added value. The Early-Stage Digital Health Investment Tool was developed to assist ministries of health in determining their readiness to introduce a digital health tool, such as an EIR, by assessing the core building blocks of digital health [12]. In practice, country health systems with sufficient readiness are likely those that have already achieved relatively high vaccination coverage. In these contexts, improved vaccination coverage may not be the primary goal of introducing EIRs.

\section{Objective}

The aims of this study are to (1) conceptualize additional ways that EIRs can add value to immunization programs and (2) assess the feasibility and potential value-add using Tanzania as a case study.

\section{Methods}

\section{Overview}

This study comprised 2 sequential phases. First, a comprehensive list of ways EIRs can potentially add value for immunization programs was developed through stakeholder interviews. Second, the added value was evaluated using Tanzania Immunization Registry (TImR) data for a prioritized subset of program needs.

\section{Phase 1}

\section{Conceptual Framework}

A comprehensive list of common barriers that country immunization programs face in achieving coverage and equity goals was used to identify the ways in which EIRs can add value. The list was adapted from a July 2019 Gavi workshop on Improving Data use in Immunization in which approximately 40 participants from the Gavi Secretariat, core and extended partners, and country representatives identified and categorized barriers. For each common barrier, the study team (EC, TKR, and LW) identified ways that EIRs could help address the barrier based on their expertise and implementation experience.

\section{Data Collection and Analysis}

Stakeholder interviews were conducted to refine the framework of the immunization program barriers and potential EIR solutions. Stakeholders were purposively selected based on their expertise in research, policy, or implementation of EIRs. A total of 7 stakeholders participated in semistructured web-based interviews facilitated by the study team (EC) from November 2019 to January 2020. A total of 4 stakeholders were government officials from countries in Sub-Saharan Africa, identified through the BID (Better Immunization Data) Learning Network [13]. A total of 3 stakeholders were from international public health agencies, donors, or implementing organizations. Summary notes from the interviews were used to refine the conceptual framework. A follow-up web-based survey (using SurveyMonkey, Momentive, Inc) was sent to a wider group of EIR experts (including interviewees) in January 2020, asking respondents to prioritize topics from the conceptual framework for further analyses. Survey responses from 17 individuals were used, in conjunction with the interview data, to prioritize the 4 topics for phase 2 analyses. 


\section{Phase 2}

\section{Setting}

Data from Tanzania's EIR were analyzed to illustrate how an EIR can add value to each of the prioritized topic areas. The Government of Tanzania partnered with the BID Initiative, funded by the Bill \& Melinda Gates Foundation and launched in 2013, to design and implement a package of solutions to improve immunization data quality and use [14]. An EIR was an essential component of the solution package. EIR design began in 2014 and went through iterations culminating in TImR, which is built on the OpenIZ platform (now known as SanteDB, SanteSuite) [15]. The Government of Tanzania has led a staged rollout of TImR to facilities across districts and regions. As of December 31, 2020, TImR was rolled out to 3736 facilities across 15 of 25 regions in mainland Tanzania and included 1.6 million client records.

\section{Data Sources}

Immunization, facility, and patient data were extracted from the TImR system with permission from the Government of Tanzania. Data were deidentified after extraction, and all analyses were conducted using deidentified data. The development and implementation of the TImR system have been discussed in detail elsewhere [15-17]. Population density data were extracted at the ward level from WorldPop's United Nations-adjusted GeoTIFFs at $100 \times 100 \mathrm{~km}$ spatial resolution using Database of Global Administrative Areas (GADM) administrative shapefiles and matched to facilities based on facility geocodes $[18,19]$. Subject matter experts were consulted on the construction of analysis variables (eg, missed opportunities for vaccination [MOVs]). Data were processed and analyzed using Alteryx (version 2020.3; Alteryx, Inc), R (version 4.0.0; R Foundation for Statistical Computing), Tableau (version 2020.2; Tableau Software, Inc), and STATA (version 14.2; StataCorp LLC).

\section{Ethics Approval}

This study received nonresearch determination from the PATH. The Government of Tanzania and the PATH have data-sharing permissions in place that guided the use of TImR data for this study.

\section{Data Restrictions}

The analyses focused on services provided between 2017 and 2019 and the vaccine doses that were included in the official Tanzania vaccine schedule, specifically Bacillus Calmette-Guérin (BCG); oral polio vaccine (OPV); diphtheria, tetanus, pertussis, hepatitis $\mathrm{B}$, and Haemophilus influenzae type b (Penta); pneumococcal conjugate vaccine (PCV); rotavirus (Rota); and measles-containing vaccine (Table 1). Data were further restricted to doses received while the TImR system was live (ie, doses logged in the system at the time of service or shortly afterward). Doses retroactively entered to complete patient medical records were included in the continuum of care analysis only. This analysis included back-entered doses for patients with at least one live TImR entry to capture the full picture of their vaccine history. A total of 3 regions (Mtwara, Rukwa, and Ruvuma) with $<50$ visits recorded in TImR by December 31, 2019, were excluded. In addition, patient IDs with $>3$ instances of a given vaccine dose (eg, OPV-1) were assumed to be dummy patient IDs used to log vaccinations provided during mass immunization campaigns and were excluded from the analysis. Patient IDs with up to 3 instances of a given dose were assumed to result from data entry errors.

Table 1. Tanzania vaccine schedule. ${ }^{\mathrm{a}}$

\begin{tabular}{lll}
\hline Vaccine dose & Scheduled visit number & Age eligibility \\
\hline BCG $^{\mathrm{b}}-0$ and $\mathrm{OPV}^{\mathrm{c}}-0$ & 1 & Birth or first contact \\
OPV-1, Penta $^{\mathrm{d}}-1, \mathrm{PCV}^{\mathrm{e}}-1$, and Rota \\
f-1 & 2 & 6 weeks \\
OPV-2, Penta-2, PCV-2, and Rota-2 & 3 & 10 weeks \\
OPV-3, Penta-3, and PCV-3 & 4 & 14 weeks \\
$\mathrm{MCV}^{\mathrm{g}}-1$ & 5 & 9 months \\
$\mathrm{MCV}-2$ & 6 & 18 months \\
\hline
\end{tabular}

${ }^{a}$ Inactivated polio vaccine immunization was excluded from our analyses as it was introduced partway through the analysis period. It would normally be received during visit 4 at the age of 14 weeks.

${ }^{\mathrm{b}}$ BCG: Bacillus Calmette-Guérin.

${ }^{\mathrm{c}} \mathrm{OPV}$ : oral polio vaccine.

${ }^{\mathrm{d}}$ Penta: diphtheria, tetanus, pertussis, hepatitis $\mathrm{B}$, and Haemophilus influenzae type b.

${ }^{\mathrm{e}} \mathrm{PCV}$ : pneumococcal conjugate vaccine.

${ }^{\mathrm{f}}$ Rota: rotavirus.

${ }^{\mathrm{g}} \mathrm{MCV}$ : measles-containing vaccine. 


\section{Definitions}

\section{Outcomes}

\section{Missed Opportunities for Vaccination}

MOVs were assessed at the visit level using the World Health Organization (WHO) definition: "any contact with health services by an individual (child or person of any age) who is eligible for vaccination (e.g., unvaccinated or partially vaccinated and free of contraindications to vaccination), which does not result in the person receiving one or more of the vaccine doses for which he or she is eligible" [20]. Dose eligibility was based on patient age, prior doses received, and time since the last dose of the vaccine sequence (if applicable). The MOV variable was constructed both as a binary (any MOV in a visit or not) and count (number of vaccine-specific MOVs per visit). Binary coding was used for the regression models, which motivated the use of logistic regression.

\section{Vaccine Dropout}

Vaccine-specific dropout for multidose vaccines was defined as receiving the first but not the last dose in the vaccine schedule (eg, receiving PCV-1 but not PCV-3). OPV dropout was defined as receiving OPV-0 or OPV-1 and not OPV-3; children who did not receive OPV-0 by the age of 2 were eligible for OPV- 1 without receiving OPV-0; therefore, either vaccine can be treated as the starting dose. We also assessed dropout between birth doses and first follow-up visit, defined as receiving either of the birth doses (BCG or OPV-0) but none of the first follow-up visit doses (OPV-1, Penta-1, PCV-1, and Rota-1). Finally, we assessed overall dropout, which is defined as receiving at least one scheduled vaccine dose but not completing the full 14-dose schedule. The dropout variables were constructed as binaries (meeting criteria for dropout or not), motivating the use of logistic regression in the models.

\section{Assigned Facility and Nonassigned Visits}

Children are assigned a home facility when they are registered in the TImR system based on their preferences and where they plan to receive care. A nonassigned visit is a visit to any health facility other than the assigned visit. This variable was constructed as a binary variable (visit at home facility or not), motivating the use of logistic regression in the models.

\section{Predictors}

\section{Dose Timeliness}

A dose was considered timely if it was received within 7 days of the scheduled date (Table 1). In practice, in Tanzania, a child is generally considered a defaulter after 7 days past their scheduled date.

\section{Urbanicity}

A facility was designated as urban if the ward in which it is located had a population density of at least 500 persons per square $\mathrm{km}$ and rural if otherwise [21]. A patient was assumed to live in an urban area if their assigned facility, presumably near their residence, was designated as urban or rural, if otherwise.

\section{Stockout}

Facility stock use, including days of 0 stock, is recorded in TImR by facility and vaccine type. Vaccine-specific stockout was defined as any period in which the stock balance for a given vaccine was zero. A composite indicator was also constructed for the proportion of days with a stockout, with the number of days with a stockout for a primary vaccine (BCG, OPV, PCV, Penta, Rota, or measles-containing vaccine $[\mathrm{MCV}])$ as the numerator and the number of days with facility stock data in the TImR system for each primary vaccine as the denominator.

\section{Age}

Age was defined in two ways: static age at the time of data extraction (December 31,2019) and age at the time of a given visit. The 2 age variables were coded into 1 -year categories up to the age of 5 years (ie, <12 months, 12-23 months, 24-35 months, 36-47 months, and 48-59 months), which is the upper limit of standard eligibility for most of the vaccines of interest.

\section{Regression Models}

For all analyses, we used mixed-effects logistic regression to assess the factors associated with the various outcomes. In all models, relevant patient and facility characteristics were included as fixed effects, and nested random intercepts for region, district, and facility ID were used to account for clustering.

\section{Results}

\section{Phase 1: EIR Added Value}

Textbox 1 lists ways that EIRs can help address common barriers faced by immunization programs in achieving coverage and equity. EIRs can add value through existing functionalities (eg, the ability to identify underimmunized children) and through functionalities that may not be a core component of existing systems (eg, the ability to serve as a platform for remote, virtual supportive supervision).

On the basis of stakeholder input, 4 topics were prioritized for phase 2 analyses:

1. Denominators and population movements, including patient movement between facilities or geographic areas for care

2. MOVs, including their frequency and any associated characteristics

3. Continuum of care, including which children drop out and when in the vaccination schedule

4. Continuous quality improvement (CQI), including trends or outliers in data quality or service delivery, to inform targeted quality improvement efforts

The remainder of this section provides an overview of the TImR data and then provides results on each of the 4 priority topic areas to illustrate how EIR data can be used to better understand denominators and population movement, MOVs, continuum of care, and CQI. 
Textbox 1. Immunization barriers and potential electronic immunization registry-based solutions.

\section{Lack of understanding about what drives immunization demand}

- EIR data can identify un- or underimmunized children and explore drivers of their vaccination status (eg, geography, demographic characteristics, and facility type).

- EIR data can be used to analyze at what point children drop out of the continuum of care.

- EIRs can have embedded decision support to guide health workers in delivering tailored messages or services to increase acceptance and uptake.

Overly complex processes

- EIRs can be designed to streamline data capture and reduce the burden of data entry.

- $\quad$ EIRs can be designed to meet decision-making needs for end users.

\section{Skill level and availability of human resources}

- Access to data through EIRs can empower and motivate users and strengthen agency.

- If EIRs are designed with individual health worker log-ins, EIRs can track human resources based on active health worker profiles.

- EIR data can identify error rates of individual health workers and link them to additional training or supportive supervision.

- $\quad$ EIRs can have embedded training resources or capacity assessments.

- EIR data can be used to forecast service delivery needs by facility or district to optimize the distribution of human resources and session times.

Geographic and social barriers to access

- EIR data can identify un- or underimmunized children to explore whether they are concentrated in certain geographic areas and if they have shared demographic characteristics to inform targeted outreach.

- EIRs can track an individual's vaccinations across public and private sector facilities.

\section{Microplanning challenges}

- EIRs can capture more accurate, timely, and complete denominators to inform microplanning.

- EIR data can be used to understand population movement or health-seeking behaviors to inform microplanning (eg, how common it is for children to move between multiple facilities).

\section{Inadequate introduction of new vaccines}

- EIR data on current vaccine delivery can be used to forecast the necessary stock and human resources to introduce new vaccines.

\section{Inadequate governance structures and capacities}

- The process of designing and introducing an EIR can help clarify and document governance structures related to immunization data.

- EIR data can provide more accurate denominator estimates to inform costing and budgeting for the EPI.

\section{A lack of resilience in leadership}

- EIRs can encourage continuous quality improvement by highlighting trends, outliers, or patterns that may require adaptive management.

- EIRs provide more timely, detailed data compared with traditional paper-based reporting, which enables timely, responsive action from leaders.

- $\quad$ EIRs can provide a platform for remote, web-based supportive supervision.

\section{Gaps in information systems}

- EIRs can show which facilities are entering data or not and factors associated with reporting.

- $\quad$ EIRs can be designed to mimic health worker workflows to streamline data collection and reporting practices.

\section{Poor quality of stock data from health facilities}

- EIR service delivery data can be triangulated to see how consistent it is with vaccine stock data and to forecast stock needs.

- $\quad$ EIR service delivery data can be used to inform decisions about vial size (eg, whether smaller vial sizes are needed in some areas to reduce waste).

\section{Poor quality of service delivery}

- EIRs can identify service delivery patterns to optimize health worker allocation and session timing to match demand.

- EIRs that capture check-in time and vaccination time can calculate patient wait times. 
- EIRs can identify missed opportunities for vaccination.

- EIRs can include stock reorder alerts to reduce stockout frequency.

\section{Vaccine safety and effectiveness}

- EIR data triangulated with patient-level data on adverse events following immunization or surveillance data can answer questions about the effectiveness of vaccines given at different times.

\section{Phase 2: Tanzania Case Study}

The sample size for the individual analyses varied because of differing inclusion criteria and missing data. In full, our sample comprised 2,444,803 vaccine doses over 958,870 visits for
559,542 patients. These visits occurred in 2359 health facilities covering 57 districts in 10 regions. The median (IQR) number of provided doses per facility per month was 40 (9-123), and the median number of visits was 17 (4-49). Table 2 provides participant demographics and facility characteristics. 
Table 2. Patient and facility characteristics. ${ }^{\text {a }}$

\begin{tabular}{|c|c|c|c|}
\hline Level and covariate & Number of visits, n (\%) & Number of patients, $\mathrm{n}(\%)$ & Number of facilities, $\mathrm{n}(\%)$ \\
\hline \multicolumn{4}{|l|}{ Patient } \\
\hline \multicolumn{4}{|l|}{ Sex } \\
\hline Female & $472,782(49.35)$ & $275,605(49.31)$ & $\mathrm{N} / \mathrm{A}^{\mathrm{b}}$ \\
\hline Male & $485,195(50.65)$ & $283,361(50.69)$ & N/A \\
\hline \multicolumn{4}{|c|}{ Age (as of December 31, 2019) } \\
\hline$<12$ months & $235,387(24.55)$ & $153,857(21.61)$ & N/A \\
\hline $12-23$ months & $300,948(31.39)$ & $183,618(25.8)$ & N/A \\
\hline 24-35 months & $300,646(31.35)$ & $143,976(20.23)$ & N/A \\
\hline $36-47$ months & $106,673(11.12)$ & $64,360(9.04)$ & N/A \\
\hline 48-59 months & $13,389(1.4)$ & $12,153(1.71)$ & N/A \\
\hline$\geq 5$ years & $1,828(0.19)$ & $153,857(21.61)$ & N/A \\
\hline \multicolumn{4}{|c|}{ Age (at time of visit; months) } \\
\hline$<12$ & $833,349(86.91)$ & N/A & N/A \\
\hline $12-23$ & $111,259(11.6)$ & N/A & N/A \\
\hline 24-35 & $10,138(1.06)$ & N/A & N/A \\
\hline $36-47$ & $2811(0.29)$ & N/A & N/A \\
\hline $48-59$ & $1283(0.13)$ & N/A & N/A \\
\hline \multicolumn{4}{|c|}{ Urbanicity (of patient) } \\
\hline Rural & $624,726(66.2)$ & $365,459(66.38)$ & N/A \\
\hline Urban & $318,972(33.8)$ & $185,106(33.62)$ & N/A \\
\hline \multicolumn{4}{|l|}{ Facility } \\
\hline \multicolumn{4}{|l|}{ Facility type } \\
\hline Dispensary & $343,525(60.39)$ & $1,953(82.79)$ & $343,525(60.39)$ \\
\hline Health center & $152,496(26.81)$ & $311(13.18)$ & $152,496(26.81)$ \\
\hline Hospital & $72,786(12.8)$ & $95(4.03)$ & $72,786(12.8)$ \\
\hline \multicolumn{4}{|c|}{ Urbanicity (of facility) } \\
\hline Rural & $621,375(65.78)$ & $364,817(65.67)$ & $1873(81.01)$ \\
\hline Urban & $323,284(34.22)$ & $190,689(34.33)$ & 439 (18.99) \\
\hline \multicolumn{4}{|c|}{$\operatorname{TImR}^{\mathrm{c}}$ use duration (as of December 31,2019 ) } \\
\hline $0-5$ months & $5038(0.53)$ & N/A & $104(4.45)$ \\
\hline 6-11 months & $282,993(29.63)$ & N/A & $1041(44.56)$ \\
\hline 1 year & $183,826(19.25)$ & N/A & $625(26.76)$ \\
\hline$\geq 2$ years & $483,201(50.59)$ & N/A & $566(24.23)$ \\
\hline \multicolumn{4}{|l|}{ Region } \\
\hline Arusha & $270,099(28.25)$ & $112,963(20.22)$ & $271(11.56)$ \\
\hline Dar es Salaam & $3062(0.32)$ & $2726(0.49)$ & $48(2.05)$ \\
\hline Dodoma & $96,059(10.05)$ & $61,033(10.93)$ & $321(13.69)$ \\
\hline Geita & $25,807(2.7)$ & $21,152(3.79)$ & $123(5.25)$ \\
\hline Kilimanjaro & $93,231(9.75)$ & $52,367(9.37)$ & $294(12.54)$ \\
\hline Lindi & $12,532(1.31)$ & $10,101(1.81)$ & $162(6.91)$ \\
\hline Morogoro & $86,815(9.08)$ & $61,697(11.04)$ & $298(12.71)$ \\
\hline
\end{tabular}




\begin{tabular}{clll}
\hline Level and covariate & Number of visits, $\mathrm{n}(\%)$ & Number of patients, $\mathrm{n}(\%)$ & Number of facilities, $\mathrm{n}(\%)$ \\
\hline Mwanza & $152,914(15.99)$ & $113,953(20.4)$ & $318(13.56)$ \\
Njombe & $11,469(1.2)$ & $9645(1.73)$ & $184(7.85)$ \\
Tanga & $204,246(21.36)$ & $113,002(20.23)$ & $326(13.9)$ \\
\hline
\end{tabular}

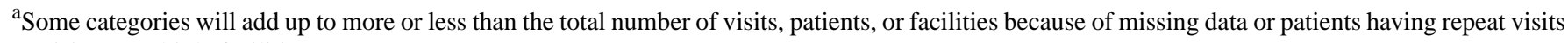
or visits at multiple facilities.

${ }^{\mathrm{b}} \mathrm{N} / \mathrm{A}$ : not applicable.

${ }^{\mathrm{c}}$ TImR: Tanzania Immunization Registry.

\section{Denominators and Population Movement}

\section{Overview}

This analysis explored population movement, that is, care seeking at alternative (nonassigned) facilities, which affects the accuracy of facility denominators. Of 810,568 total visits, $765,835(94.48 \%)$ were at a child's assigned facility, 15,575 $(1.92 \%)$ were at a nonassigned facility within $5 \mathrm{~km}$ of the child's assigned facility, 14,147 (1.82\%) at facilities located $>5 \mathrm{~km}$ from the assigned facility but within the same district, 12,267 $(1.51 \%)$ in a different district within the same region, and 2926 $(0.36 \%)$ in a different region. Figure 1 summarizes attendance by region for all visits and visits to nonassigned facilities. Although children were similarly likely to seek care at their assigned facility across regions, patterns of care seeking to nonassigned facilities varied. For example, of visits to nonassigned facilities, children in Dar Es Salaam region were most likely to seek care within $5 \mathrm{~km}(64 / 85,75 \%)$, whereas children in Geita were most likely to seek care outside of the region $(131 / 187,70.1 \%)$.

Table 3 explores visits at assigned and nonassigned facilities based on patient and assigned facility characteristics. There was little variation in the likelihood of a visit being at a nonassigned facility based on patient sex, assigned facility ownership, or assigned facility type. As expected, patients assigned to urban facilities and patients whose assigned facility had a higher number of facilities within $5 \mathrm{~km}$ were more likely to visit nonassigned facilities. Older children were more likely to visit nonassigned facilities; however, this could be an artifact of older children having more visits (and thus more opportunities to visit other facilities) or being more likely to have moved since being entered into the TImR system (ie, no longer residing near their assigned facility). Patients assigned to facilities newer to the TImR system were less likely to visit nonassigned facilities.

Figure 1. Visits to assigned and nonassigned facilities.

\section{All visits}

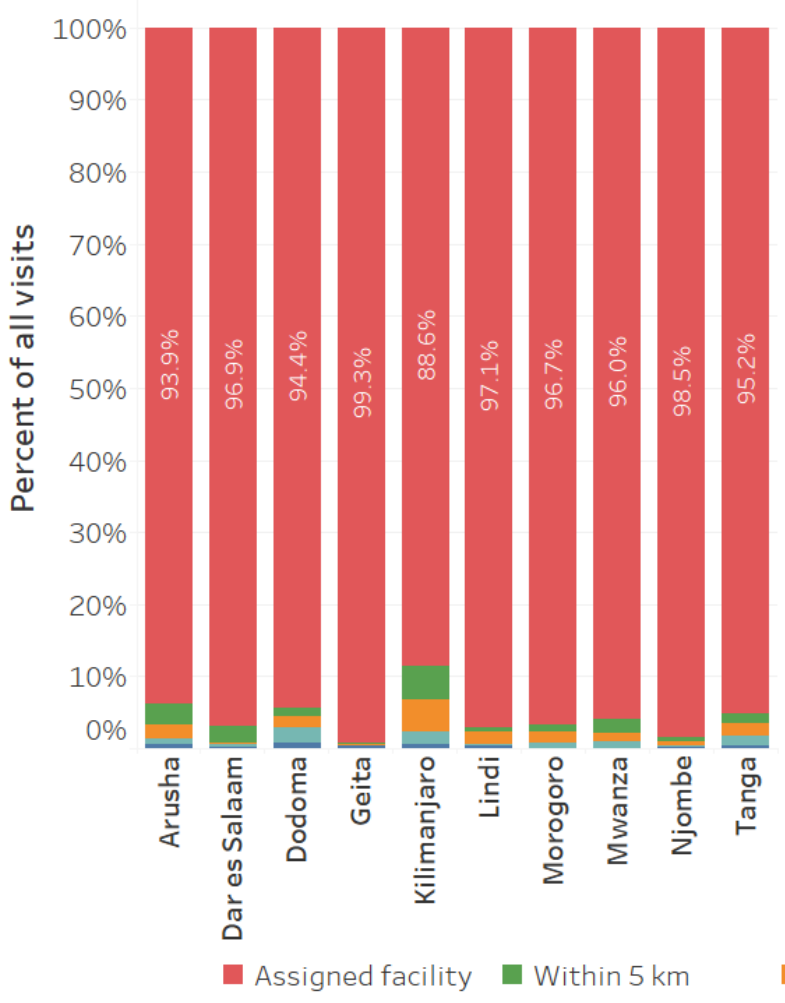

Visits to a nonassigned facility

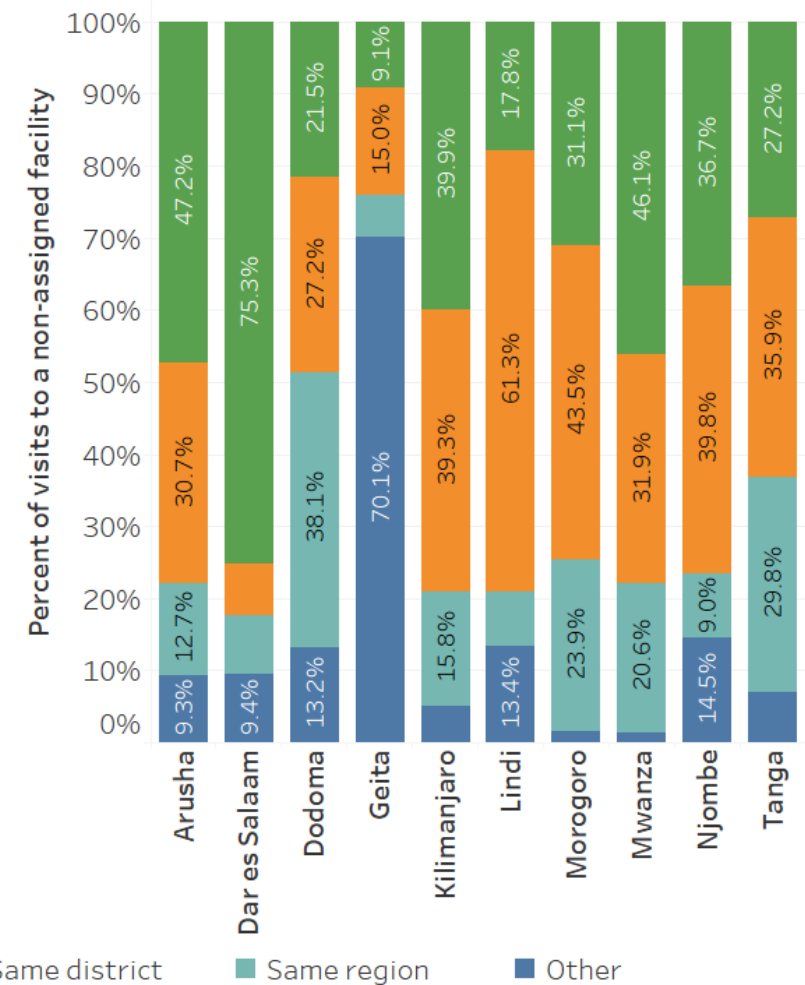


Table 3. Visits to assigned and nonassigned facilities by patient and assigned facility characteristics $(\mathrm{N}=810,568)$.

\begin{tabular}{|c|c|c|c|}
\hline Covariate & Total visits, $\mathrm{n}$ & At nonassigned facility, $\mathrm{n}(\%)$ & At assigned facility, n (\%) \\
\hline \multicolumn{4}{|l|}{ Sex } \\
\hline Female & 400,507 & $22,028(5.5)$ & $378,479(94.5)$ \\
\hline Male & 409,164 & $22,504(5.5)$ & $386,660(94.5)$ \\
\hline \multicolumn{4}{|c|}{ Age at time of visit (months) } \\
\hline$<12$ & 232,653 & $10,935(4.7)$ & $221,718(95.3)$ \\
\hline $12-23$ & 296,680 & $16,317(5.5)$ & $280,363(94.5)$ \\
\hline $24-35$ & 220,613 & $12,796(5.8)$ & $207,817(94.2)$ \\
\hline $36-47$ & 53,190 & $3989(7.5)$ & 49,201 (92.5) \\
\hline $48-59$ & 5908 & $520(8.8)$ & $5388(91.2)$ \\
\hline \multicolumn{4}{|c|}{ Assigned facility type } \\
\hline Dispensary & 490,965 & $25,530(5.2)$ & $465,435(94.8)$ \\
\hline Health center & 223,344 & $13,401(6.0)$ & $209,943(94.0)$ \\
\hline Hospital & 96,259 & $5679(5.9)$ & $90,580(94.1)$ \\
\hline \multicolumn{4}{|c|}{ Assigned facility urbanicity } \\
\hline Rural & 598,804 & $25,150(4.2)$ & $573,654(95.8)$ \\
\hline Urban & 205,070 & $19,482(9.5)$ & $185,588(90.5)$ \\
\hline \multicolumn{4}{|c|}{ Assigned facility ownership } \\
\hline Private & 148,088 & $9922(6.7)$ & $138,166(93.3)$ \\
\hline Public & 655,786 & $34,101(5.2)$ & $621,685(94.8)$ \\
\hline \multicolumn{4}{|c|}{ Assigned facility $\operatorname{TImR}^{\mathrm{a}}$ duration (at time of visit) } \\
\hline $0-5$ months & 461,325 & $20,760(4.5)$ & $440,565(95.5)$ \\
\hline 6-11 months & 196,127 & $14,513(7.4)$ & $181,614(92.6)$ \\
\hline 1 year & 133,746 & $8693(6.5)$ & $125,053(93.5)$ \\
\hline$\geq 2$ years & 19,370 & $697(3.6)$ & $18,673(96.4)$ \\
\hline \multicolumn{4}{|c|}{ Number of facilities within $5 \mathrm{~km}$ of assigned facility } \\
\hline 0 & 277,803 & $9167(3.3)$ & $268,636(96.7)$ \\
\hline 1 & 157,322 & $7551(4.8)$ & $149,771(95.2)$ \\
\hline $2-5$ & 171,457 & $13,888(8.1)$ & $157,569(91.9)$ \\
\hline$>5$ & 192,290 & $20,767(10.8)$ & $171,523(89.2)$ \\
\hline
\end{tabular}

${ }^{\mathrm{a}}$ TImR: Tanzania Immunization Registry.

\section{Spatial Variation in Assigned Facility Attendance}

Figure 2 shows the proportion of all visits by children assigned to a given facility that occurred at the assigned facility. Facilities with low attendance appeared to cluster in northern Kilimanjaro, southeastern Arusha, southeastern and urban Mwanza, and coastal and central Tanga. 
Figure 2. Proportion of visits at assigned facilities by facility geocode.

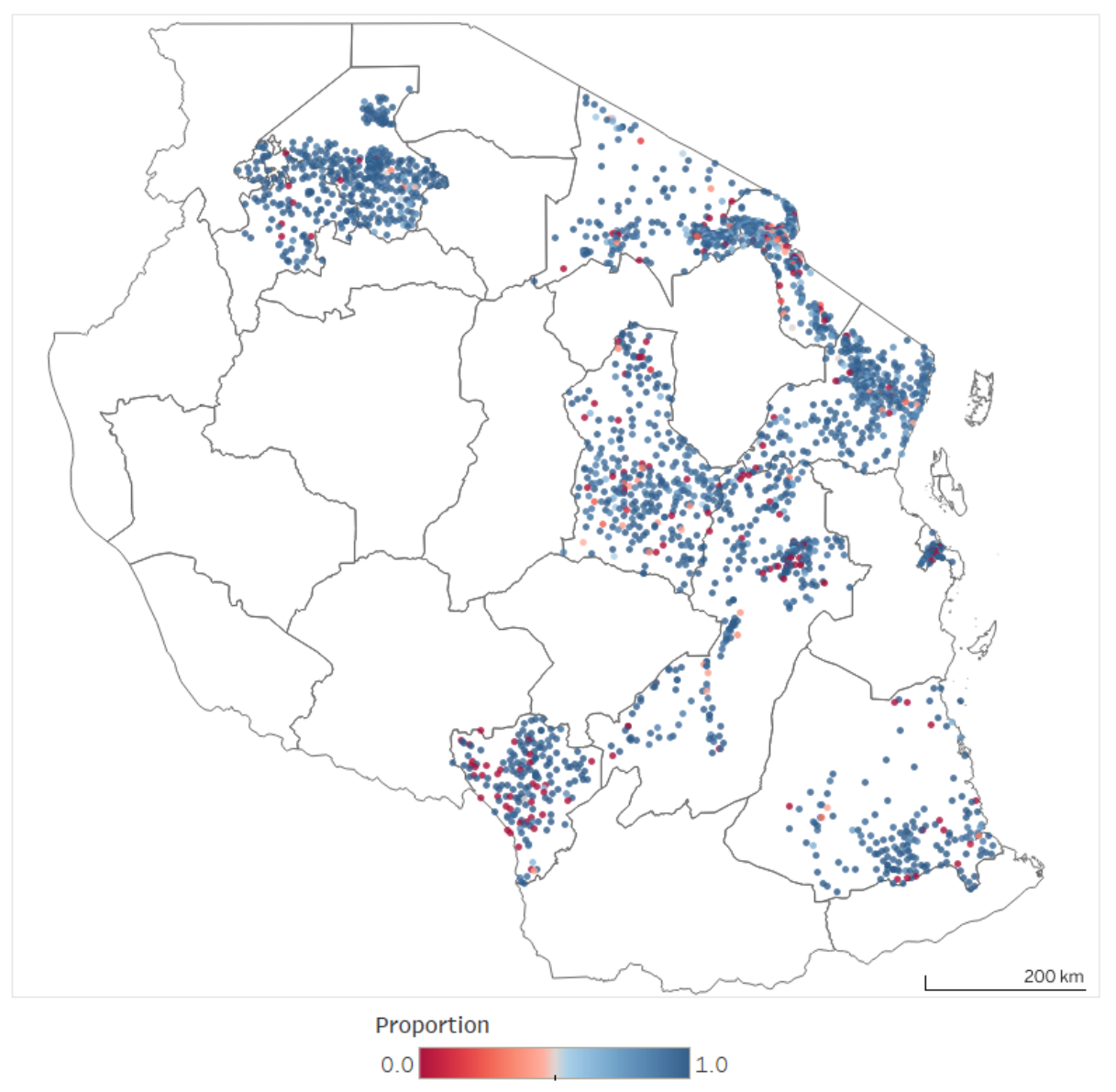

\section{Model Results}

Table 4 shows results from the logistic regression model with a given visit to a nonassigned facility as the outcome of interest. Children assigned to public facilities and health centers or hospitals, facilities with a longer duration of TImR use, and facilities in areas with higher health facility density were significantly more likely to visit a nonassigned facility. Children attending facilities with a greater number of recorded visits were significantly less likely to visit a nonassigned facility. Interestingly, the relationship with age was no longer monotonic after adjusting for other covariates. As compared with children aged $\leq 12$ months, children aged 1 to 2 years were less likely to visit a nonassigned facility, whereas those aged 3 to 4 years were more likely. 
Table 4. Population movement regression model results.

\begin{tabular}{|c|c|c|c|c|}
\hline \multirow[t]{2}{*}{ Covariate } & \multicolumn{2}{|l|}{ Unadjusted model } & \multicolumn{2}{|l|}{ Adjusted model } \\
\hline & $\mathrm{OR}^{\mathrm{a}}(95 \% \mathrm{CI})$ & $P$ value & $\mathrm{aOR}^{\mathrm{b}}(95 \% \mathrm{CI})$ & $P$ value \\
\hline \multicolumn{5}{|l|}{ Sex } \\
\hline Female & Reference & $\mathrm{N} / \mathrm{A}^{\mathrm{c}}$ & Reference & N/A \\
\hline Male & $0.98(0.96-1.00)$ & .08 & $0.99(0.97-1.01)$ & .21 \\
\hline \multicolumn{5}{|l|}{ Age (months) } \\
\hline$<12$ & Reference & N/A & Reference & N/A \\
\hline $12-23$ & $0.71(0.69-0.74)$ & $<.001$ & $0.79(0.76-0.82)$ & $<.001$ \\
\hline $24-35$ & $0.64(0.61-0.66)$ & $<.001$ & $0.87(0.83-0.91)$ & $<.001$ \\
\hline $36-47$ & $0.85(0.81-0.89)$ & $<.001$ & $1.30(1.23-1.37)$ & $<.001$ \\
\hline $48-59$ & $1.20(1.08-1.33)$ & $<.001$ & $1.89(1.69-2.11)$ & $<.001$ \\
\hline \multicolumn{5}{|l|}{ Assigned facility urbanicity } \\
\hline Rural & Reference & N/A & Reference & N/A \\
\hline Urban & $1.42(1.11-1.82)$ & .01 & $0.93(0.74-1.18)$ & .56 \\
\hline \multicolumn{5}{|l|}{ Assigned facility ownership } \\
\hline Private & Reference & N/A & Reference & N/A \\
\hline Public & N/A & N/A & $1.37(1.13-1.66)$ & .001 \\
\hline \multicolumn{5}{|l|}{ Assigned facility type } \\
\hline Dispensary & Reference & N/A & Reference & N/A \\
\hline Health center & $1.16(0.94-1.43)$ & .17 & $1.71(1.41-2.07)$ & $<.001$ \\
\hline Hospital & $1.53(1.07-2.21)$ & .02 & $2.13(1.52-2.98)$ & $<.001$ \\
\hline Assigned facility stockout ( $\%$ of days) & $1.00(1.00-1.01)$ & .48 & $1.00(0.99-1.01)$ & .94 \\
\hline Total assigned visits (log) & $0.28(0.26-0.32)$ & $<.001$ & $0.24(0.21-0.27)$ & $<.001$ \\
\hline \multicolumn{5}{|l|}{ Assigned facility $\operatorname{TImR}^{\mathrm{d}}$ duration } \\
\hline $0-5$ months & Reference & N/A & Reference & N/A \\
\hline 6-11 months & $2.40(1.17-4.94)$ & .02 & $1.55(1.44-1.68)$ & $<.001$ \\
\hline $12-23$ months & $2.84(1.17-6.88)$ & .02 & $7.29(6.75-7.87)$ & $<.001$ \\
\hline$\geq 2$ years & $2.15(0.88-5.28)$ & .09 & $8.15(7.48-8.89)$ & $<.001$ \\
\hline \multicolumn{5}{|c|}{ Number of facilities within $5 \mathrm{~km}$ of assigned facility } \\
\hline 0 & Reference & N/A & Reference & N/A \\
\hline 1 & $1.62(1.50-1.76)$ & $<.001$ & $2.03(1.97-2.09)$ & $<.001$ \\
\hline $2-5$ & $8.38(7.75-9.05)$ & $<.001$ & $2.06(1.98-2.15)$ & $<.001$ \\
\hline$>5$ & $9.55(8.76-10.40)$ & $<.001$ & $1.48(1.35-1.64)$ & $<.001$ \\
\hline
\end{tabular}

${ }^{\mathrm{a} O R}$ : odds ratio.

$\mathrm{b}_{\mathrm{aOR}}$ : adjusted odds ratio.

${ }^{\mathrm{c}} \mathrm{N} / \mathrm{A}$ : not applicable.

${ }^{\mathrm{d}}$ TImR: Tanzania Immunization Registry.

\section{Missed Opportunities for Vaccination}

\section{Overview}

MOVs, where the patient did not receive at least one vaccine for which they were eligible, were observed in $23.69 \%$ $(226,525 / 956,195)$ of visits. Although we found little variation in the likelihood of an MOV based on sex, there was notable heterogeneity across age groups, facility urbanicity, facility type, and duration of TImR use at the facility (Table 5). The higher likelihood of an MOV among younger patients may be an artifact of the higher number of scheduled doses in the first year of life, and therefore, greater opportunity for missed doses. 
Table 5. Visits with missed opportunities for vaccination (MOVs) by vaccine type and patient and facility characteristics. ${ }^{\mathrm{a}}$

\begin{tabular}{|c|c|c|c|c|c|c|c|c|}
\hline \multirow[t]{2}{*}{ Covariate } & \multirow{2}{*}{$\begin{array}{l}\text { Number } \\
\text { of visits }\end{array}$} & \multicolumn{7}{|c|}{ Visits with an MOV by vaccine, $\mathrm{n}(\%)$} \\
\hline & & Any vaccine & Penta ${ }^{b}$ & $\mathrm{OPV}^{\mathrm{c}}$ & $\mathrm{BCG}^{\mathrm{d}}$ & $\mathrm{MCV}^{\mathrm{e}}$ & $\operatorname{Rota}^{\mathrm{f}}$ & $\mathrm{PCV}^{\mathrm{g}}$ \\
\hline Overall & 956,195 & $226,525(23.69)$ & $60,364(6.31)$ & $58,040(6.07)$ & $54,924(5.74)$ & $5781(0.60)$ & $95,651(10.00)$ & $63,684(6.66)$ \\
\hline \multicolumn{9}{|l|}{ Sex } \\
\hline Female & 471,406 & $111,636(23.68)$ & $29,692(6.30)$ & $28,570(6.06)$ & $27,234(5.78)$ & $2794(0.59)$ & $47,011(9.97)$ & $31,358(6.65)$ \\
\hline Male & 483,896 & $114,582(23.68)$ & $30,594(6.32)$ & $29,409(6.08)$ & $27,583(5.70)$ & $2953(0.61)$ & $48,488(10.02)$ & $32,236(6.66)$ \\
\hline \multicolumn{9}{|c|}{ Age group (months) } \\
\hline$<12$ & 831,018 & $215,576(25.94)$ & $55,453(6.67)$ & $53,492(6.44)$ & $51,461(6.19)$ & $2080(0.25)$ & $95,651(11.51)$ & $58,627(7.05)$ \\
\hline $12-23$ & 110,968 & $9298(8.38)$ & $4376(3.94)$ & $4027(3.63)$ & $2913(2.63)$ & $2973(2.68)$ & $-\mathrm{h}$ & $4492(4.05)$ \\
\hline $24-35$ & 10,123 & $1239(12.24)$ & $374(3.69)$ & $350(3.46)$ & $354(3.5)$ & $609(6.02)$ & - & $417(4.12)$ \\
\hline $36-47$ & 2805 & $293(10.45)$ & $114(4.06)$ & $125(4.46)$ & $145(5.17)$ & $83(2.96)$ & - & $104(3.71)$ \\
\hline $48-59$ & 1281 & $119(9.29)$ & $47(3.67)$ & $46(3.59)$ & $51(3.98)$ & $36(2.81)$ & - & $44(3.43)$ \\
\hline \multicolumn{9}{|l|}{ Visited facility type } \\
\hline Dispensary & 563,186 & $138,714(24.63)$ & $38,917(6.91)$ & $34,295(6.09)$ & $30,271(5.37)$ & $3646(0.65)$ & $61,852(10.98)$ & $43,948(7.80)$ \\
\hline Health center & 270,290 & $58,821(21.76)$ & $14,359(5.31)$ & $16,463(6.09)$ & $15,233(5.64)$ & $1465(0.54)$ & $22,830(8.45)$ & $14,035(5.19)$ \\
\hline Hospital & 122,719 & $28,990(23.62)$ & $7088(5.78)$ & $7282(5.93)$ & $9420(7.68)$ & $670(0.55)$ & $10,969(8.94)$ & $5701(4.65)$ \\
\hline \multicolumn{9}{|c|}{ Visited facility urbanicity } \\
\hline Rural & 620,214 & $156,768(25.28)$ & $41,729(6.73)$ & $41,956(6.76)$ & $35,968(5.8)$ & $4178(0.67)$ & $67,467(10.88)$ & $47,301(7.63)$ \\
\hline Urban & 322,199 & $65,247(20.25)$ & $16,588(5.15)$ & $14,540(4.51)$ & $18,082(5.61)$ & $1540(0.48)$ & $26,318(8.17)$ & $15,165(4.71)$ \\
\hline \multicolumn{9}{|c|}{ Visited facility TImR ${ }^{i}$ duration (at time of visit) } \\
\hline $0-5$ months & 600,234 & $130,595(21.76)$ & $23,894(3.98)$ & $37,593(6.26)$ & $36,829(6.14)$ & $2340(0.39)$ & $53,416(8.90)$ & $36,747(6.12)$ \\
\hline 6-11 months & 197,188 & $50,634(25.68)$ & $18,569(9.42)$ & $11,582(5.87)$ & $10,329(5.24)$ & $1321(0.67)$ & $21,660(10.98)$ & $12,598(6.39)$ \\
\hline 1 year & 135,342 & $37,630(27.8)$ & $15,231(11.25)$ & $7277(5.38)$ & $5864(4.33)$ & $1827(1.35)$ & $17,584(12.99)$ & $11,410(8.43)$ \\
\hline$\geq 2$ years & 19,705 & $6576(33.37)$ & $2430(12.33)$ & $1140(5.79)$ & $1188(6.03)$ & $248(1.26)$ & $2871(14.57)$ & $2641(13.40)$ \\
\hline \multicolumn{9}{|c|}{ Visited facility stockout (\% days) } \\
\hline$<10 \%$ & 666,531 & $155,134(23.27)$ & $40,379(6.06)$ & $41,754(6.26)$ & $34,730(5.21)$ & $3990(0.60)$ & $66,733(10.01)$ & $46,524(6.98)$ \\
\hline $10 \%-19 \%$ & 153,392 & $36,516(23.81)$ & $9888(6.45)$ & 8543 (5.57) & $10,200(6.65)$ & $960(0.63)$ & $15,191(9.90)$ & $8672(5.65)$ \\
\hline $20 \%-29 \%$ & 77,730 & $21,007(27.03)$ & $6297(8.10)$ & $4226(5.44)$ & 7103 (9.14) & $490(0.63)$ & 7957 (10.24) & $4291(5.52)$ \\
\hline$\geq 30 \%$ & 54,072 & $12,203(22.57)$ & $2998(5.54)$ & $3031(5.61)$ & $2683(4.96)$ & $306(0.57)$ & $5090(9.41)$ & 3552 (6.57) \\
\hline
\end{tabular}

${ }^{a}$ Vaccine-specific percentages do not add up to the total missed opportunity for vaccination (MOV) percentage as patients can have MOVs for multiple vaccine types in a single visit.

${ }^{\mathrm{b}}$ Penta: diphtheria, tetanus, pertussis, hepatitis $\mathrm{B}$, and Haemophilus influenzae type $\mathrm{b}$.

${ }^{\mathrm{c}} \mathrm{OPV}$ : oral polio vaccine.

${ }^{\mathrm{d}}$ BCG: Bacillus Calmette-Guérin.

${ }^{\mathrm{e}} \mathrm{MCV}$ : measles-containing vaccine.

${ }^{\mathrm{f}}$ Rota: rotavirus.

${ }^{\mathrm{g}} \mathrm{PCV}$ : pneumococcal conjugate vaccine.

${ }^{\mathrm{h}}$ Children are not considered eligible for rotavirus immunization after the first year of life.

${ }^{\mathrm{i}}$ TImR: Tanzania Immunization Registry.

Of the 557,674 children included in the analysis, 167,115 (29.97\%) had $\geq 1$ MOVs. The mean number of MOVs per child was 0.61 (SD 1.20). Among the 167,115 children with an MOV, 85,697 (51.28\%) had $\geq 1$ MOV (range 1-15). Of 338,439 recorded MOVs, rotavirus was the most likely to have an MOV (accounting for $28.26 \%$ of all MOVs; $n=95,650)$, followed by PCV (18.82\%, 63,682), Penta (17.84\%, 60,363), OPV (17.15\%, $58,039), \operatorname{BCG}(16.23 \%, 54,924)$, and MCV $(1.71 \%, 5781)$. The lower MOV proportion for MCV was likely because of fewer 
visits where children were age-eligible for MCV (aged at least 9 months).

\section{MOV Reasons}

The TImR system allows providers to indicate the reasons why a scheduled and eligible dose was not provided. However, the reason will only be noted if a dose is knowingly not given and thus is absent for doses for which providers did not recognize the patient's eligibility. For eligible doses that the provider logged as missed, the data indicated the mechanisms behind MOVs.

Table 6 details MOV reasons by vaccine type. Of 338,439 recorded MOVs, 183,623 (54.26\%) had a listed reason: 177,624
$(52.48 \%)$ were because of facility stockout, $2474(0.73 \%)$ because of medical contraindication, $3184(0.94 \%)$ because of being late (generally meant to indicate that the child is too old to start the vaccine sequence), $178(0.05 \%)$ because of guardian refusal, and $163(0.05 \%)$ because of expired stock. These reasons varied by vaccine type, with roughly three-quarters of Penta and PCV MOVs because of stockout but less than half for $\mathrm{BCG}, \mathrm{MCV}$, and rotavirus MOVs. Rotavirus MOVs were more likely to result from medical contraindications (913/95,650, 0.95\%) compared with MOVs of the other vaccine types, whereas MCV had the highest likelihood of being missed because of guardian refusal $(15 / 5781,0.26 \%)$.

Table 6. Reasons for missed opportunities for vaccination (MOVs).

\begin{tabular}{llllllll}
\hline Vaccine type & $\begin{array}{l}\text { Number of } \\
\text { recorded MOVs }\end{array}$ & \multicolumn{2}{l}{ MOV reason (MOVs for given vaccine type), n (\%) } & & & \\
& & Stockout & Medical contraindication & Late & Refusal & Expired stock & No reason provided \\
\hline Overall & 338,439 & $177,624(52.48)$ & $2474(0.73)$ & $3184(0.94)$ & $178(0.05)$ & $163(0.05)$ & $154,816(45.74)$ \\
Rota $^{\mathrm{a}}$ & 95,650 & $34,315(35.88)$ & $913(0.95)$ & $761(0.8)$ & $34(0.04)$ & $38(0.04)$ & $59,589(62.3)$ \\
OPV $^{\mathrm{b}}$ & 58,039 & $37,056(63.85)$ & $296(0.51)$ & $1118(1.93)$ & $37(0.06)$ & $31(0.05)$ & $19,501(33.6)$ \\
Penta $^{\mathrm{c}}$ & 60,363 & $46,133(76.43)$ & $309(0.51)$ & $558(0.92)$ & $36(0.06)$ & $62(0.1)$ & $13,265(21.98)$ \\
PCV $^{\mathrm{d}}$ & 63,682 & $47,712(74.92)$ & $434(0.68)$ & $834(1.31)$ & $39(0.06)$ & $37(0.06)$ & $14,626(22.97)$ \\
$\mathrm{BCG}^{\mathrm{e}}$ & 54,924 & $24,430(44.48)$ & $126(0.23)$ & $513(0.93)$ & $40(0.07)$ & $11(0.02)$ & $29,804(54.26)$ \\
$\mathrm{MCV}^{\mathrm{f}}$ & 5781 & $2694(46.6)$ & $12(0.21)$ & $117(2.02)$ & $15(0.26)$ & $5(0.09)$ & $2938(50.82)$ \\
\hline
\end{tabular}

${ }^{\mathrm{a}}$ Rota: rotavirus.

${ }^{\mathrm{b}} \mathrm{OPV}$ : oral polio vaccine.

${ }^{\mathrm{c}}$ Penta: diphtheria, tetanus, pertussis, hepatitis B, and Haemophilus influenzae type b.

${ }^{\mathrm{d}} \mathrm{PCV}$ : pneumococcal conjugate vaccine.

${ }^{\mathrm{e}} \mathrm{BCG}$ : Bacillus Calmette-Guérin.

${ }_{\mathrm{f}} \mathrm{MCV}$ : measles-containing vaccine.

\section{Model Results}

Results from the any-vaccine MOV and OPV-specific MOV models were selected as illustrative examples of interest and are shown in Table 7. Unadjusted results can be found in Multimedia Appendices 1 and 2. Age group and TImR duration were significantly associated with any MOV and OPV-specific MOVs. Compared with children aged $<1$ year, older children were substantially less likely to experience MOVs in both models. This may be because of the greater opportunity for MOVs at younger ages because of more scheduled doses in the first year of life. Interestingly, TImR use duration at the time of visit showed opposite directionality between the models, with longer TImR implementation associated with a higher likelihood of any MOV but lower likelihood of OPV-specific MOVs, suggesting that there may be different mechanisms leading to MOVs by vaccine type. 
Table 7. Missed opportunity for vaccination (MOV) regression model results.

\begin{tabular}{|c|c|c|c|c|}
\hline \multirow[t]{2}{*}{ Covariate } & \multicolumn{2}{|l|}{ Any MOV } & \multicolumn{2}{|l|}{$\mathrm{OPV}^{\mathrm{a}} \mathrm{MOV}$} \\
\hline & $\mathrm{aOR}^{\mathrm{b}}(95 \% \mathrm{CI})$ & $P$ value & $\mathrm{aOR}(95 \% \mathrm{CI})$ & $P$ value \\
\hline \multicolumn{5}{|l|}{ Sex } \\
\hline Female & Reference & $N / A^{c}$ & Reference & N/A \\
\hline Male & $1.00(0.99-1.01)$ & .90 & $1.00(0.98-1.02)$ & .88 \\
\hline \multicolumn{5}{|l|}{ Age (months) } \\
\hline $0-11$ & Reference & N/A & Reference & N/A \\
\hline $12-23$ & $0.19(0.18-0.19)$ & $<.001$ & $0.41(0.40-0.43)$ & $<.001$ \\
\hline $24-35$ & $0.25(0.23-0.26)$ & $<.001$ & $0.33(0.29-0.37)$ & $<.001$ \\
\hline $36-47$ & $0.19(0.17-0.22)$ & $<.001$ & $0.40(0.33-0.49)$ & $<.001$ \\
\hline $48-59$ & $0.18(0.15-0.22)$ & $<.001$ & $0.33(0.24-0.46)$ & $<.001$ \\
\hline \multicolumn{5}{|l|}{ Urbanicity } \\
\hline Rural & Reference & N/A & Reference & N/A \\
\hline Urban & $0.90(0.75-1.08)$ & .25 & $0.96(0.73-1.26)$ & .78 \\
\hline \multicolumn{5}{|l|}{ Ownership } \\
\hline Private & Reference & N/A & Reference & N/A \\
\hline Public & $1.02(0.88-1.18)$ & .85 & $0.89(0.71-1.12)$ & .31 \\
\hline \multicolumn{5}{|l|}{ Facility type } \\
\hline Dispensary & Reference & N/A & Reference & N/A \\
\hline Health center & $0.89(0.77-1.03)$ & .11 & $1.13(0.91-1.40)$ & .28 \\
\hline Hospital & $0.99(0.76-1.28)$ & .91 & $0.92(0.62-1.36)$ & .68 \\
\hline \multicolumn{5}{|c|}{ Facility $\operatorname{TImR}^{\mathrm{d}}$ duration (at time of visit) } \\
\hline $0-5$ months & Reference & N/A & Reference & N/A \\
\hline 6-11 months & $1.61(1.58-1.63)$ & $<.001$ & $0.90(0.88-0.93)$ & $<.001$ \\
\hline $12-23$ months & $2.27(2.22-2.31)$ & $<.001$ & $0.73(0.71-0.76)$ & $<.001$ \\
\hline$\geq 2$ years & $3.15(3.03-3.27)$ & $<.001$ & $0.67(0.62-0.72)$ & $<.001$ \\
\hline
\end{tabular}

${ }^{\mathrm{a} O P V}$ : oral polio vaccine.

$\mathrm{b}_{\mathrm{aOR}}$ : adjusted odds ratio.

${ }^{\mathrm{c}} \mathrm{N} / \mathrm{A}$ : not applicable.

${ }^{\mathrm{d}}$ TImR: Tanzania Immunization Registry.

\section{Continuum of Care}

This analysis explored the vaccine dropout. To ensure common eligibility for doses, this analysis was restricted to children aged 12 to 47 months at the end of 2019 and focused on the 14 doses scheduled for the first year of life (ie, through MCV-1; Table $1)$.

\section{Immunization Coverage}

Overall, 93,619 (31.79\%) of 294,464 children in our sample were fully immunized for doses scheduled in the first year of life (inclusive of OPV-0), with a further 39,718 (13.48\%) receiving all scheduled doses, except for MCV-1. Figure 3 shows the doses received and timeliness by vaccine type and dose. As expected, there was a drop-off in coverage with later doses in each vaccine sequence. Timeliness also decreased monotonically with later doses in a sequence. 
Figure 3. Vaccine coverage and dose timeliness. BCG: Bacillus Calmette-Guérin; MCV: measles-containing vaccine; OPV: oral polio vaccine; PCV: pneumococcal conjugate vaccine.

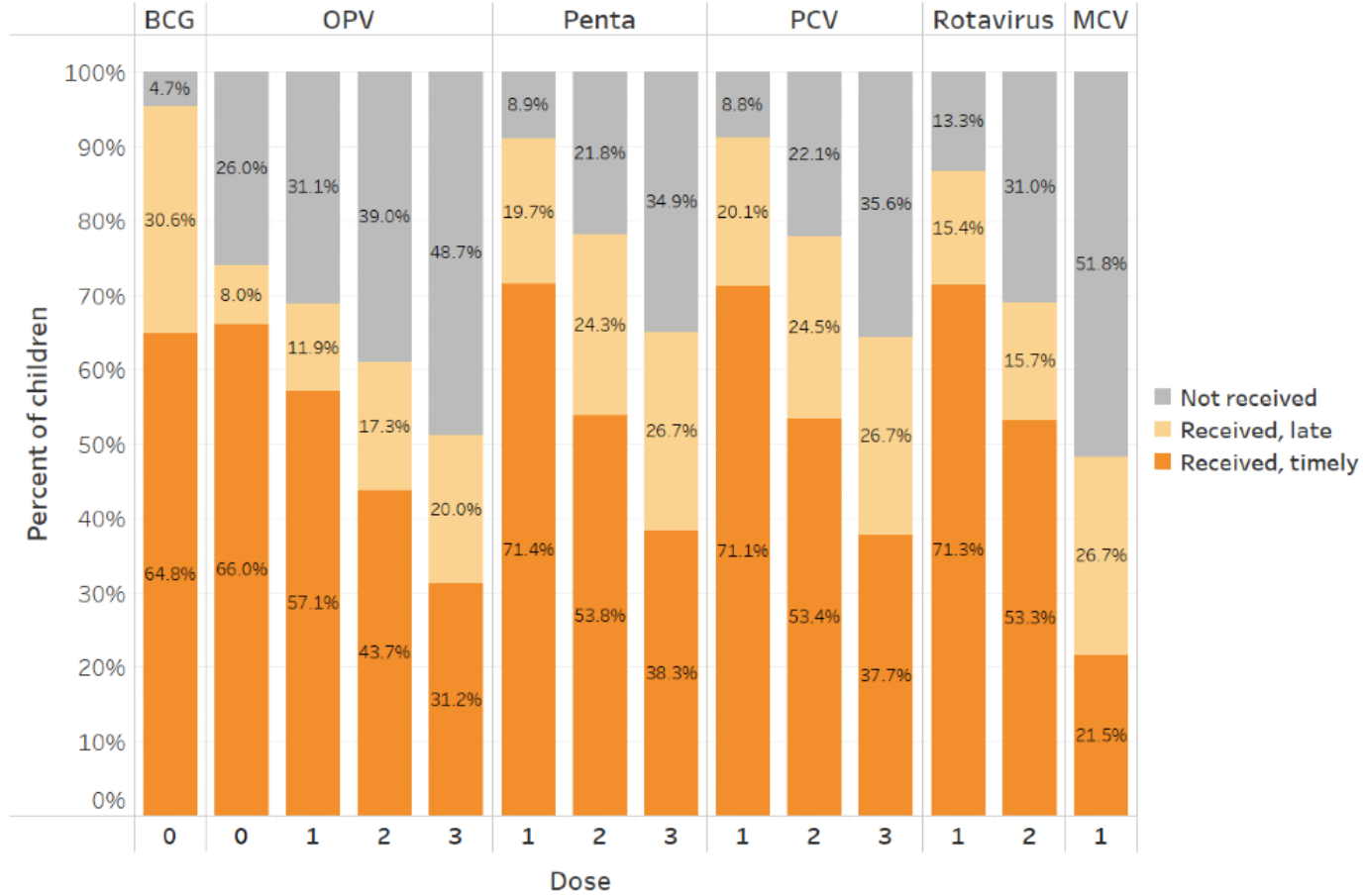

\section{Vaccine Dropout}

Table 8 details dropout rates across patient characteristics. For multidose vaccine-specific dropout, OPV had the highest rate $(66,798 / 217796,30.67 \%)$, followed by PCV $(78,767 / 268,582$, $29.33 \%)$, Penta $(76,659 / 268,315,28.57 \%)$, and Rota $(52,086 / 255,337,20.4 \%)$. Rotavirus may have had a lower dropout rate because there were only 2 doses in the sequence. There were common trends for all outcomes, such as older children and private facilities showing lower levels of dropout for all types of dropouts. However, some trends were outcome-/vaccine-specific, such as rural facilities showing higher levels of dropout for all vaccines except OPV. Most of these differences were marginal except for dropout by age group. 
Table 8. Dropout by patient and facility characteristics.

\begin{tabular}{|c|c|c|c|c|c|c|}
\hline \multirow[t]{2}{*}{ Covariate } & \multicolumn{6}{|c|}{ Children dropped out, n (\%) } \\
\hline & Penta $^{\mathrm{a}}$ & $\mathrm{OPV}^{\mathrm{b}}$ & $\operatorname{Rota}^{\mathrm{c}}$ & $\mathrm{PCV}^{\mathrm{d}}$ & Birth or first & Overall dropout \\
\hline Overall & $76,659(28.57)$ & $66,798(30.67)$ & $52,086(20.4)$ & 78,767 (29.33) & $16,414(5.79)$ & $194,765(66.14)$ \\
\hline \multicolumn{7}{|l|}{ Sex } \\
\hline Female & $37,591(28.47)$ & 32,735 (30.6) & $25,547(20.31)$ & $38,652(29.23)$ & $8073(5.78)$ & $95,793(66.1)$ \\
\hline Male & $38,962(28.66)$ & 33,955 (30.69) & $26,466(20.45)$ & $40,006(29.4)$ & $8262(5.75)$ & $98,633(66.14)$ \\
\hline \multicolumn{7}{|c|}{ Age group (months) } \\
\hline $12-23$ & 51,057 (31.9) & $46,743(34.42)$ & $34,951(22.84)$ & $52,795(32.87)$ & $12,159(6.99)$ & 124,599 (69.99) \\
\hline $24-35$ & $25,602(23.65)$ & $20,055(24.46)$ & $17,135(16.75)$ & $25,972(24.06)$ & 4255 (3.88) & $70,166(60.26)$ \\
\hline \multicolumn{7}{|c|}{ Assigned facility type } \\
\hline Health center & $21,375(29.52)$ & $20,176(33.41)$ & $14,236(20.62)$ & $21,790(30.06)$ & $5161(6.74)$ & $54,369(68.01)$ \\
\hline Hospital & 8451 (30.69) & 7885 (35.68) & $5634(21.77)$ & $8562(31.15)$ & $2915(9.63)$ & $22,117(70.55)$ \\
\hline \multicolumn{7}{|c|}{ Assigned facility urbanicity } \\
\hline Rural & $51,651(28.88)$ & $41,372(30.23)$ & 35,904 (21.19) & $53,075(29.68)$ & $10,448(5.54)$ & $131,395(67)$ \\
\hline Urban & $23,375(27.46)$ & 23,778 (30.94) & $15,051(18.44)$ & $24,077(28.25)$ & $5711(6.34)$ & $59,872(64.1)$ \\
\hline \multicolumn{7}{|c|}{ Assigned facility ownership } \\
\hline Private & $14,514(30.83)$ & $11,976(32.64)$ & 9272 (20.78) & 14,567 (30.89) & $3072(6.16)$ & $35,856(68.61)$ \\
\hline Public & $61,289(28.03)$ & $53,867(30.13)$ & $42,184(20.26)$ & 63,223 (28.9) & $13,186(5.71)$ & $156,847(65.54)$ \\
\hline
\end{tabular}

${ }^{a}$ Penta: diphtheria, tetanus, pertussis, hepatitis B, and Haemophilus influenzae type b.

${ }^{\mathrm{b}} \mathrm{OPV}$ : oral polio vaccine.

${ }^{\mathrm{c}}$ Rota: rotavirus.

${ }^{\mathrm{d}} \mathrm{PCV}$ : pneumococcal conjugate vaccine.

\section{Immunization Typologies}

To better understand vaccination profiles, we constructed immunization archetypes using all possible combinations of eligible scheduled doses. Patients were fit into these archetypes based on their immunization history. Table 9 shows the 10 most common archetypes by vaccine doses received. After being fully vaccinated and fully vaccinated except for MCV-1, the third most common typology was receiving all doses except for the OPV sequence $(19,322 / 294,464,6.56 \%$ of children), followed by dropping out between the second and third visits $(13,270 / 294,464,4.51 \%)$ or between the third and fourth visits $(13,102 / 294,464,4.45 \%)$, and receiving only the birth doses (BCG and OPV-0; 10,156/294,464, 3.45\%). 
Table 9. Immunization typologies (10 most common).

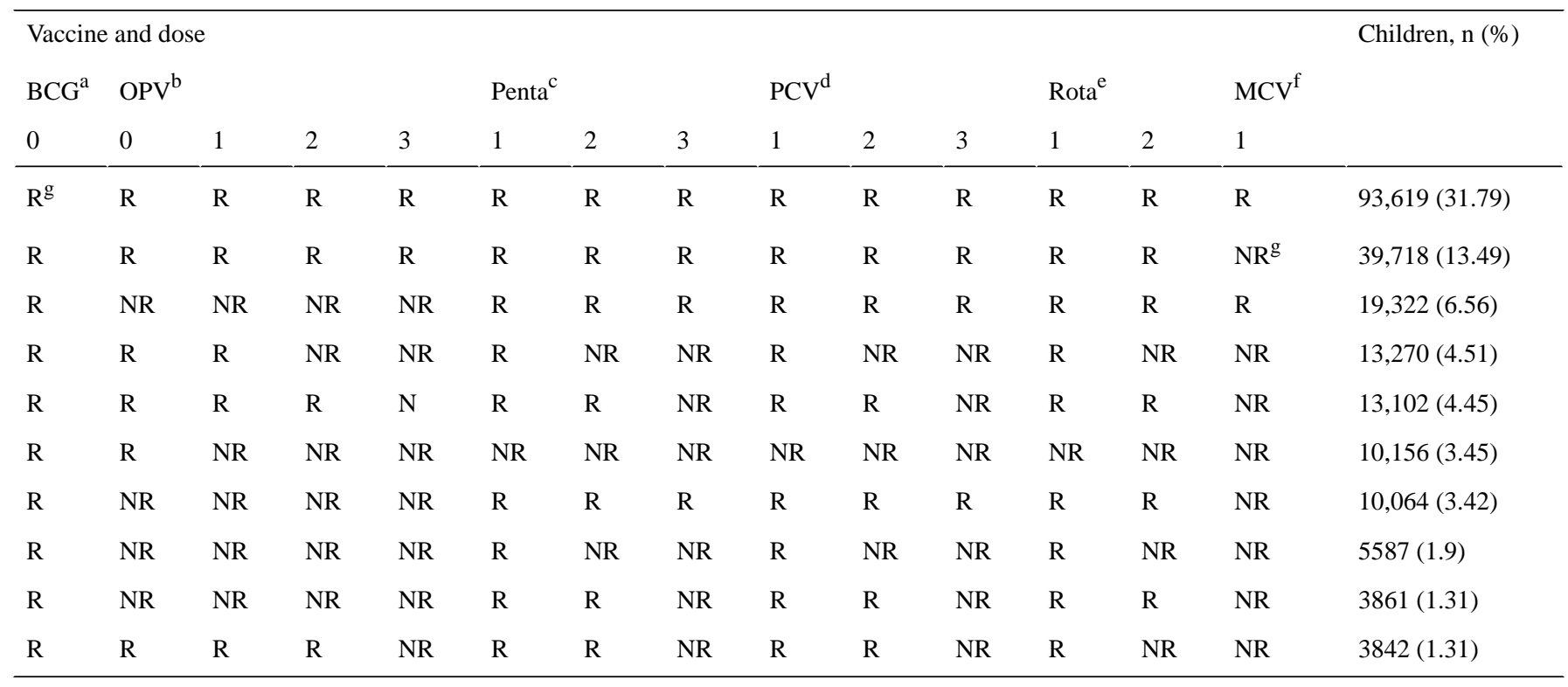

${ }^{\mathrm{a} B C G}$ : Bacillus Calmette-Guérin.

${ }^{\mathrm{b}} \mathrm{OPV}$ : oral polio vaccine.

${ }^{\mathrm{c}}$ Penta: diphtheria, tetanus, pertussis, hepatitis $\mathrm{B}$, and Haemophilus influenzae type $\mathrm{b}$.

${ }^{\mathrm{d}} \mathrm{PCV}$ : pneumococcal conjugate vaccine.

${ }^{\mathrm{e}}$ Rota: rotavirus.

${ }_{\mathrm{f}}^{\mathrm{MCV} \text { : measles-containing vaccine. }}$

$\mathrm{g}_{\text {" }} \mathrm{R}$ " indicates a given dose was received, while "NR" indicates the dose was not received.

\section{Visit Dropout}

To understand dropout between different scheduled visits, we analyzed the proportion of children that had received any vaccine from each of the 5 scheduled touchpoints with the immunization system in the first year of life (Table 1). Overall, $96.29 \%(283,548 / 294,464)$ of children received at least one of the birth doses (BCG or OPV-0), 93.16\% (274,314/294,464) received at least one of the visit 2 doses (OPV-1, PCV-1, Penta-1, or Rota-1), 80.98\% $(238,450 / 294,464)$ received at least one visit 3 dose, $67.52 \%(198,812 / 294,464)$ received at least one visit 4 dose, and $48.21 \%(141,948 / 294,464)$ received the visit 5 dose.

\section{Model Results}

The results from the Penta and overall dropout models were selected as illustrative examples of interest and are shown below. Unadjusted results can be found in Multimedia Appendices 3 and 4. As shown in Table 10, older age was significantly associated with a lower likelihood of both Penta and overall dropout (ie, starting but not finishing the 14-dose schedule). In the overall dropout model, urban facilities were associated with a significantly lower likelihood of overall dropout, and public facilities were associated with a higher likelihood. These trends were not observed in the Penta dropout model. 
Table 10. Dropout regression model results.

\begin{tabular}{|c|c|c|c|c|}
\hline \multirow[t]{2}{*}{ Covariate } & \multicolumn{2}{|l|}{ Penta dropout } & \multicolumn{2}{|l|}{ Overall dropout } \\
\hline & $\mathrm{aOR}^{\mathrm{a}}(95 \% \mathrm{CI})$ & $P$ value & $\mathrm{aOR}(95 \% \mathrm{CI})$ & $P$ value \\
\hline \multicolumn{5}{|l|}{ Sex } \\
\hline Female & Reference & $\mathrm{N} / \mathrm{A}^{\mathrm{b}}$ & Reference & N/A \\
\hline Male & $1.02(1.00-1.04)$ & .06 & $1.01(0.99-1.03)$ & .21 \\
\hline \multicolumn{5}{|l|}{ Age (months) } \\
\hline $12-23$ & Reference & N/A & Reference & N/A \\
\hline $24-35$ & $0.23(0.22-0.23)$ & $<.001$ & $0.19(0.19-0.19)$ & $<.001$ \\
\hline \multicolumn{5}{|l|}{ Assigned facility urbanicity } \\
\hline Rural & Reference & N/A & Reference & N/A \\
\hline Urban & $0.86(0.71-1.04)$ & .11 & $0.83(0.70-0.99)$ & .03 \\
\hline \multicolumn{5}{|l|}{ Assigned facility ownership } \\
\hline Private & Reference & N/A & Reference & N/A \\
\hline Public & $1.04(0.90-1.22)$ & .57 & $1.15(1.00-1.33)$ & .047 \\
\hline \multicolumn{5}{|l|}{ Assigned facility type } \\
\hline Dispensary & Reference & N/A & Reference & N/A \\
\hline Health center & $0.95(0.81-1.10)$ & .49 & $1.04(0.91-1.22)$ & .58 \\
\hline Hospital & $1.19(0.91-1.54)$ & .20 & $1.27(1.00-1.61)$ & .06 \\
\hline Assigned facility stockout ( $\%$ of days) & $1.00(1.00-1.01)$ & .12 & $1.00(1.00-1.01)$ & .28 \\
\hline
\end{tabular}

$\mathrm{a}_{\mathrm{aOR}}$ : adjusted odds ratio.

${ }^{\mathrm{b}} \mathrm{N} / \mathrm{A}$ : not applicable.

\section{Continuous Quality Improvement}

EIRs provide data for the rapid assessment of CQI improvement measures. These assessments can help improve service provision by identifying areas in need of targeted training or other quality improvement interventions. As shown in Table 11, $10 \%$ of facilities account for most of the issues, suggesting that targeted interventions to identified facilities could greatly improve care.
These results use absolute numbers and, therefore, will be biased toward facilities with higher patient loads and longer TImR implementation durations. In practice, CQI analyses would likely be restricted to specific months or quarters, reducing any duration bias. Absolute figures can also offer greater efficiency by targeting CQI interventions to providers or facilities with the highest absolute number of issues.

Table 11. Continuous quality improvement.

\begin{tabular}{llll}
\hline All facilities $(\mathrm{n}=2345)$ & $\begin{array}{l}\text { Issues accounted for, } \mathrm{n}(\%) \\
\text { Visits to a nonassigned facility } \\
\end{array}$ & $\begin{array}{l}\text { Visits with an } \mathrm{MOV}^{\mathrm{b}} \\
(\mathrm{n}=44,733)\end{array}$ & $\begin{array}{l}\text { (any vaccine; }>\mathrm{n}=226,525) \\
\text { Children who have dropped out } \\
\text { (full dropout; } \mathrm{n}=194,765)^{\mathrm{a}}\end{array}$ \\
\hline $10 \%(\mathrm{n}=134)$ & $36,307(81.16)$ & $126,226(55.72)$ & $112,895(57.96)$ \\
$25 \%(\mathrm{n}=586)$ & $42,937(95.99)$ & $215,989(95.35)$ & $159,584(81.94)$ \\
$50 \%(\mathrm{n}=1172)$ & $44,715(99.96)$ & $225,569(99.58)$ & $188,281(96.67)$ \\
$75 \%(\mathrm{n}=1758)$ & $44,733(100)$ & & $193,971(99.59)$ \\
\hline
\end{tabular}

a Aggregated by child's assigned facility.

${ }^{b}$ MOV: missed opportunity for vaccination.

\section{Discussion}

\section{Principal Findings}

EIRs can add value in multiple ways. Access to individual-level data that captures all touchpoints with the immunization program allows for new analyses that can benefit immunization programs, national and regional ministry staff, health care providers and administrators, funders, and other stakeholders [8,9,22]. Descriptive statistics can be used to rapidly monitor service provision and vaccination coverage or inform quality improvement efforts. Longitudinal and spatial analyses can be 
used to understand temporospatial changes in care and coverage. Risk factor analyses can be used to identify patient and facility characteristics associated with immunization issues (eg, dropout). These analyses can be targeted to the relevant stakeholder groups. For example, facility-level statistics for a given district can inform targeted supportive supervision, and national-level coverage trends can enable evidence-based policy development. EIRs also allow for more cost-effective and rapid synthesis of immunization data; many of these descriptive statistics and analyses would not be possible using the aggregate data available in the routine health information system or would require significant additional funding, time, and other resources for survey data collection [9]. The analyses presented in this study are intended to illustrate the types of insights that EIR data can provide to immunization programs.

\section{Denominators and Population Movement}

Inaccurate population denominators are a common challenge for monitoring coverage, improving implementation, and informing planning, such as projecting vaccine stock and staffing needs. A recent scoping review of immunization data quality in LMICs found that denominators were often inaccurate, infrequently adjusted, and inconsistent between the district and national levels [23]. Population denominators are influenced by migration, urbanization, and refugee crises, among other population dynamics that can have large effects at the local level [24]. Population denominators are further complicated by children seeking care at different facilities over time. The Strategic Advisory Group of Experts working group on the quality and use of global immunization and surveillance data identified inaccurate denominators as a common challenge and noted the lack of guidance on how to improve the accuracy of denominators and track mobile populations [25].

EIRs greatly simplify tracking patients who seek care at multiple facilities, enabling a more nuanced understanding of population movement across both geography and time and allowing for more robust coverage estimates. The use of the TImR data allowed us to explore both the magnitude of and factors associated with seeking care at facilities other than the patient's assigned, or home, facility. Our analysis revealed that a small subset of patients sought care outside their assigned facility (44,733/810,568, 5.52\%); however, this varied by region; facility urbanicity, type, ownership, patient volume, and duration of TImR system use; density of facilities in the immediate area; and patient age. In addition, where patients seeking care varied by region, patients in some regions were more likely to travel to other districts and regions for care. For example, children who did not attend their assigned facility in Geita were most likely to attend a facility outside of the region, potentially because of population mobility associated with mining in the region. These insights can help inform resource allocation. EIRs also greatly simplify tracking patients who seek care at multiple facilities, decreasing the likelihood of missed or redundant doses. Although a small number of children in the Tanzania case study sought care outside their assigned facilities, some areas would have a much larger nomadic or mobile population. For example, in Cameroon, children born at home, immigrants, emigrants, and nomadic populations are not accurately accounted for when planning outreach vaccination sessions, which contributes to delaying or not vaccinating an estimated $30 \%$ to $70 \%$ of the population in some districts [26].

\section{Missed Opportunities for Vaccination}

Identifying and avoiding MOVs is an important and cost-effective method for achieving greater vaccination coverage. The challenge is in identifying when, where, and among which children or facilities MOVs are experienced to address them. Integration of clinical decision support systems within EIRs can automate the determination of child dose eligibility and alert the provider, which has been shown to reduce MOVs for routine childhood immunizations [27,28]. In addition, by collating vaccination history with child and facility characteristics, EIRs naturally allow for exploration of MOVs across these characteristics and by different vaccines. Our analysis of the TImR data showed that MOVs were highest among children aged $<12$ months (as mentioned, potentially because of the higher number of scheduled doses in the first year of life); however, there were few significant differences by other individual or facility characteristics. Other studies of countries in Sub-Saharan Africa have identified additional demographic and socioeconomic characteristics associated with increased odds of MOVs, including high birth order, high number of under 5 children in the house, lack of maternal education, lack of media access, and household and neighborhood poverty [29,30]. Although these data were not captured in TImR, they could be captured by an EIR to enable new analyses and equity insights [8,31].

This information can be used by providers to identify children who may be at higher risk of experiencing an MOV. In addition, it can be used by managers to identify providers and facilities with higher rates of MOVs for supportive supervision or refresher training or identify areas with high rates of vaccine hesitancy for outreach campaigns. In addition, EIRs can provide insight into the mechanisms behind MOVs, such as stock issues and vaccine-specific hesitancy. Where data were available, stockouts were the primary reason for MOVs, whereas mechanisms such as vaccine hesitancy and medical contraindications were relatively rare. The TImR data also showed that rotavirus was the most likely to have an MOV, which may indicate that eligibility requirements should be reviewed or refresher training provided. For additional insights, analysis of EIR data can be complemented by other tools such as those included in the WHO MOV strategy toolkit [20].

\section{Continuum of Care}

Identifying where in the vaccine schedule some children drop out and why they drop out is another key challenge for achieving high levels of vaccine coverage. Understanding which vaccine doses and child and facility characteristics are associated with failure to complete a vaccine sequence or the full vaccine schedule can help inform service provision, training, and quality improvement measures at the facility, regional, and national levels. In the TImR data, nearly half of children aged 12 to 35 months were fully vaccinated or had received all doses except MCV-1 of the 14-dose under-12-month schedule (ie, through MCV-1). Among children who did not complete the vaccine schedule, levels of dropout varied by vaccine. Facility characteristics associated with dropout also varied by vaccine; 
for example, assigned facility urbanicity was significantly associated with a lower likelihood of overall dropout (ie, starting but not finishing the 14-dose schedule) but not Penta-specific dropout, suggesting that the mechanisms behind dropout may vary by vaccine. Continuum of care analyses could be further expanded if the EIR data were linked to a birth registration system. In the Tanzania case study, 5.79\% $(16,414 / 283,548)$ of children dropped out between birth and the first immunization dose; however, this may be an underestimation if some children are not registered at birth. In countries with a strong civil registration and vital statistics system, linking the EIR to birth registration or an antenatal care registry could expand the continuum of care analysis. Using EIRs to explore immunization typologies can also provide insight into which vaccines and visits require greater care. For example, in the TImR data, $6.56 \%$ $(19,322 / 294,464)$ of children were fully vaccinated through MCV-1 except for the 3 to 4 OPV doses, highlighting the need for greater research into barriers to OPV coverage.

\section{CQI Analysis}

The CQI analysis showed that most issues (eg, MOVs) came from a minority of facilities. EIRs enable decision-makers at the national and subnational levels to quickly assess and identify providers, facilities, or geographic areas for targeted quality improvement measures, thereby improving the quality of care and increasing improvement in intervention effectiveness.

\section{Added Value of EIRs}

These analyses were designed to show the potential of EIRs to allow for a more nuanced, rapid, and cost-effective evaluation of vaccine program data to facilitate data use for decision-making. For example, automated dashboards of key indicators (eg, vaccine-specific coverage, stockouts, and child dropout) can inform planning and clinical practice at the facility level without the need for on-site data analysis. Providers can also use EIRs to simplify the tracking of individual patients, particularly those seeking care at multiple facilities, to improve the quality of care and reduce issues such as MOVs [27,28]. The integration of EIRs with SMS text messaging services to automate appointment and outreach to children at risk of defaulting has been shown to reduce dropout rates for routine childhood immunizations [4]. At the district and regional levels, the evaluation of underperforming facilities can be used for targeted supportive supervision and supplemental training. At the national level, up-to-date data on geographic and spatial trends in vaccine coverage can be used to inform nationwide campaigns, resource allocation, or policy development.

Designed well, EIRs can democratize immunization data. However, they require the necessary support to function effectively. The Early-Stage Digital Health Investment Tool has identified 6 building blocks for effective digital health systems: human capacity, investments and funding, data capture and use, infrastructure, standards and interoperability, and governance and policy [12]. Strong building blocks can maximize the effectiveness of EIR systems; however, this can pose a challenge in some low- and middle-income settings where 1 or several of these building blocks may be lacking. The WHO estimates that $50 \%$ of low-income and $24 \%$ of lower-middle-income countries have strong institutional capacity or involvement in data analysis at the national ministry [22]. With technical capacity often centralized at the national level, these figures are even lower at the subnational level. Furthermore, $54 \%$ of low-income and $41 \%$ of lower-middle-income countries are rated nascent, limited, or moderate in their capacity to have data and evidence drive policy and planning [22]. Implementing robust and routinized data frameworks, including EIRs, can address gaps in data availability and provide mechanisms to harness the data to drive evidence-based policy and planning. Automation and tailoring of data output to specific end users (eg, facility-level indicator dashboards for providers) can simplify data analysis and interpretation. However, effective use of EIR data for decision-making will require health care workers and administrators at all levels to have the skills, motivation, and autonomy to understand and act on the data $[16,17]$. Leadership at the national and regional levels should prioritize capacity building to enable the health system to make use of EIR data [32].

EIR is a solution that aims to improve immunization program performance. The efficiency and impact of EIRs can be maximized by introducing them in combination with other interventions, such as capacity strengthening for data use, vaccine stock management systems, data governance frameworks, or SMS text messaging reminders for caregivers. Interventions that use multiple mechanisms to address various barriers to data use have been found to be more successful in achieving immunization data use and action [33].

\section{Limitations}

The TImR results are intended to illustrate the ways EIRs can add value to immunization programs by providing actionable information for health care providers and managers. The results are not intended to be generalizable to Tanzania as a whole because of several data limitations. First, regions and districts implemented TImR at various points in time, meaning that some geographies are over- or underrepresented in the results. Second, and relatedly, only a subset of regions in Tanzania have introduced TImR; therefore, immunization services delivered outside the TImR coverage area are not captured in the results. Third, children who may live within the TImR coverage area but have not had a touchpoint with the immunization delivery system (also known as zero dose children) or were not registered at birth are not captured in the results. Fourth, this study did not assess data completeness, and any incomplete data (eg, providers not entering all immunizations into TImR) may limit the accuracy of the results. Fifth, prior studies of the TImR data have shown reduced system use over time, potentially biasing results toward facilities with greater capability to maintain reporting systems [16]. Sixth, as mentioned earlier, the analyses were limited to data captured in TImR. Although these data can be powerful for diagnosing issues, they do not capture all patient, facility, or geographic characteristics that may influence immunization delivery and can be limited in explaining trends. For example, MOVs may be underestimated as this study only captures MOVs during immunization visits and not during nonimmunization visits [34,35]. As noted earlier, some characteristics shown to be associated with MOVs were not captured in TImR. Triangulation with other data sources or 
targeted follow-up data collection can help answer the why questions. Finally, the lens used in this study was the assessment of the potential added value of EIRs. This study does not attempt to highlight the challenges associated with implementing or maintaining EIRs, although many such challenges have been identified elsewhere $[1,3,8,9,17,36]$.

\section{Conclusions}

EIRs have the potential to add substantial value to immunization stakeholders at all levels of the health system beyond measuring vaccine coverage. Individual-level data captured through EIRs can enable new analyses to understand immunization service delivery or care-seeking patterns, potential risk factors for underimmunization, and where challenges occur. Notably, most issues (eg, occurrence of MOVs, visits to a nonassigned facility, and number of defaulters) occur in a minority of facilities, highlighting the potential for EIRs to inform targeted quality improvement efforts. However, to achieve this potential, country programs need to leverage and strengthen their capacity for collecting, analyzing, and interpreting the data. Measures and analyses should be prioritized to match the needs and capabilities of the immunization program. Ideally, the prioritized measures should be integrated into routine systems to facilitate ongoing CQI efforts. As EIRs are introduced and scaled in LMICs, implementers and researchers should continue to share real-world examples and build an evidence base for how EIRs can add value to immunization programs, particularly for innovative uses.

\section{Acknowledgments}

The authors thank the Ministry of Health in Tanzania, particularly the staff in the Immunization and Vaccines Development program, for their insights on the analyses and use of the Tanzania Immunization Registry data. The authors also thank the stakeholders who shared their insights and expertise through interviews and web-based surveys. Finally, the authors would like to thank the Bill \& Melinda Gates Foundation for providing support for this study.

\section{Conflicts of Interest}

TKR provided funding for this research in her role as a senior program officer at the Bill \& Melinda Gates Foundation.

\section{Multimedia Appendix 1}

Complete missed opportunity for vaccination (any vaccine) regression model results.

[DOCX File, 16 KB-Multimedia Appendix 1]

\section{Multimedia Appendix 2}

Complete missed opportunity for vaccination (oral polio vaccine) regression model results.

[DOCX File, 17 KB-Multimedia Appendix 2]

\section{Multimedia Appendix 3}

Complete dropout (Penta) regression model results.

[DOCX File, 16 KB-Multimedia Appendix 3]

\section{Multimedia Appendix 4}

Complete dropout (overall) regression model results.

[DOCX File, 15 KB-Multimedia Appendix 4]

\section{References}

1. Danovaro-Holliday MC, Contreras MP, Pinto D, Molina-Aguilera IB, Miranda D, García O, et al. Assessing electronic immunization registries: the Pan American Health Organization experience. Rev Panam Salud Publica 2019;43:e28 [FREE Full text] [doi: 10.26633/RPSP.2019.28] [Medline: 31093252]

2. Freeman VA, DeFriese GH. The challenge and potential of childhood immunization registries. Annu Rev Public Health 2003;24:227-246. [doi: 10.1146/annurev.publhealth.24.100901.140831] [Medline: 12668757]

3. Namageyo-Funa A, Samuel A, Bloland P, Macneil A. Considerations for the development and implementation of electronic immunization registries in Africa. Pan Afr Med J 2018;30:81 [FREE Full text] [doi: 10.11604/pamj.2018.30.81.11951] [Medline: $\underline{30344865]}$

4. Nguyen NT, Vu HM, Dao SD, Tran HT, Nguyen TX. Digital immunization registry: evidence for the impact of mHealth on enhancing the immunization system and improving immunization coverage for children under one year old in Vietnam. Mhealth 2017;3:26 [FREE Full text] [doi: 10.21037/mhealth.2017.06.03] [Medline: 28828373]

5. Chandir S, Siddiqi DA, Dharma VK, Shah MT, Turab A, Khan MI, et al. Zindagi Mehfooz (Safe Life) digital immunization registry: leveraging low-cost technology to improve immunization coverage and timeliness in Pakistan. iproc 2018 Sep 17;4(2):e11770. [doi: $10.2196 / 11770]$ 
6. Uddin MJ, Shamsuzzaman M, Horng L, Labrique A, Vasudevan L, Zeller K, et al. Use of mobile phones for improving vaccination coverage among children living in rural hard-to-reach areas and urban streets of Bangladesh. Vaccine 2016 Jan 4;34(2):276-283. [doi: 10.1016/j.vaccine.2015.11.024] [Medline: 26647290]

7. Groom H, Hopkins DP, Pabst LJ, Morgan JM, Patel M, Calonge N, Community Preventive Services Task Force. Immunization information systems to increase vaccination rates: a community guide systematic review. J Public Health Manag Pract 2015;21(3):227-248. [doi: 10.1097/PHH.0000000000000069] [Medline: 24912082]

8. Pan American Health Organization. Electronic Immunization Registry : Practical Considerations for Planning, Development, Implementation, and Evaluation. Washington, DC: PAHO; 2017.

9. Dolan SB, Carnahan E, Shearer JC, Beylerian EN, Thompson J, Gilbert SS, et al. Redefining vaccination coverage and timeliness measures using electronic immunization registry data in low- and middle-income countries. Vaccine 2019 Mar 22;37(13):1859-1867 [FREE Full text] [doi: 10.1016/j.vaccine.2019.02.017] [Medline: 30808566]

10. Mvundura M, Di Giorgio L, Vodicka E, Kindoli R, Zulu C. Assessing the incremental costs and savings of introducing electronic immunization registries and stock management systems: evidence from the better immunization data initiative in Tanzania and Zambia. Pan Afr Med J 2020;35(Suppl 1):11 [FREE Full text] [doi: 10.11604/pamj.supp.2020.35.1.17804] [Medline: 32373262]

11. Mvundura M, Di Giorgio L, Lymo D, Mwansa FD, Ngwegwe B, Werner L. The costs of developing, deploying and maintaining electronic immunisation registries in Tanzania and Zambia. BMJ Glob Health 2019;4(6):e001904 [FREE Full text] [doi: 10.1136/bmjgh-2019-001904] [Medline: $\underline{31803511]}$

12. Gagnaire K. EDIT: a tool for the greater good internet. Kati Collective. 2020. URL: https://www.linkedin.com/pulse/ edit-tool-greater-good-kirsten-gagnaire/ [accessed 2021-12-16]

13. Better Immunization Data (BID) initiative. BID Learning Network. 2021. URL: https://bidinitiative.org/bln/ [accessed 2021-12-17]

14. The BID initiative story: improving health services through innovation in data quality and use. Better Immunization Data (BID) Initiative. 2018. URL: https://bidinitiative.org/wp-content/uploads/BIDStory PRINT English R1 clickable.pdf [accessed 2021-12-17]

15. Seymour D, Werner L, Mwansa FD, Bulula N, Mwanyika H, Dube M, et al. Electronic immunization registries in Tanzania and Zambia: shaping a minimum viable product for scaled solutions. Front Public Health 2019;7:218 [FREE Full text] [doi: 10.3389/fpubh.2019.00218] [Medline: 31440494]

16. Carnahan E, Ferriss E, Beylerian E, Mwansa FD, Bulula N, Lyimo D, et al. Determinants of facility-level use of electronic immunization registries in Tanzania and Zambia: an observational analysis. Glob Health Sci Pract 2020 Sep 30;8(3):488-504 [FREE Full text] [doi: 10.9745/GHSP-D-20-00134] [Medline: $\underline{33008860]}$

17. Dolan SB, Alao ME, Mwansa FD, Lymo DC, Bulula N, Carnahan E, et al. Perceptions of factors influencing the introduction and adoption of electronic immunization registries in Tanzania and Zambia: a mixed methods study. Implement Sci Commun 2020;1:38 [FREE Full text] [doi: 10.1186/s43058-020-00022-8] [Medline: 32885195]

18. Global high resolution population denominators project. WorldPop. 2020. URL: https://www.worldpop.org/ [accessed 2021-02-03]

19. GADM database of global administrative areas, version 2. Global Administrative Areas (GADM). 2012. URL: https://gadm. org/ [accessed 2020-10-02]

20. Reducing Missed Opportunities for Vaccination (MOV). World Health Organization. URL: https://www.who.int/teams/ immunization-vaccines-and-biologicals/essential-programme-on-immunization/implementation/ reducing-missed-opportunities-for-vaccination-(mov) [accessed 2021-12-17]

21. Wineman A, Alia DY, Anderson CL. Definitions of "rural" and "urban" and understandings of economic transformation: Evidence from Tanzania. J Rural Stud 2020 Oct;79:254-268 [FREE Full text] [doi: 10.1016/j.jrurstud.2020.08.014] [Medline: 33132492]

22. World Health Organization. SCORE for health data technical package: global report on health data systems and capacity, 2020. Geneva: World Health Organization; 2021:License: CC BY-NC-SA 3.0 IGO.

23. Harrison K, Rahimi N, Danovaro-Holliday MC. Factors limiting data quality in the expanded programme on immunization in low and middle-income countries: a scoping review. Vaccine 2020 Jun 19;38(30):4652-4663 [FREE Full text] [doi: 10.1016/j.vaccine.2020.02.091] [Medline: 32446834]

24. Corrêa G, Verstraete P, Soundardjee R, Shankar M, Paterson C, Hampton L, et al. Immunization programmes and notifications of vital events. Bull World Health Organ 2019 Apr 01;97(4):306-308 [FREE Full text] [doi: 10.2471/BLT.18.210807] [Medline: 30940988]

25. Scobie HM, Edelstein M, Nicol E, Morice A, Rahimi N, MacDonald NE, SAGE Working Group on Immunization and Surveillance Data Quality and Use. Improving the quality and use of immunization and surveillance data: summary report of the working group of the strategic advisory group of experts on immunization. Vaccine 2020 Oct 27;38(46):7183-7197 [FREE Full text] [doi: 10.1016/j.vaccine.2020.09.017] [Medline: 32950304]

26. Ateudjieu J, Yakum NM, Goura AP, Guenou E, Beyala LB, Amada L, et al. Tracking demographic movements and immunization status to improve children's access to immunization (TDM-IAI): protocol for a field-based randomized controlled trial. JMIR Res Protoc 2021 Mar 08;10(2):e21734 [FREE Full text] [doi: 10.2196/21734] [Medline: 33555269] 
27. Fiks AG, Grundmeier RW, Biggs LM, Localio AR, Alessandrini EA. Impact of clinical alerts within an electronic health record on routine childhood immunization in an urban pediatric population. Pediatrics 2007 Oct;120(4):707-714 [FREE Full text] [doi: 10.1542/peds.2007-0257] [Medline: 17908756]

28. Mayne SL, duRivage NE, Feemster KA, Localio AR, Grundmeier RW, Fiks AG. Effect of decision support on missed opportunities for human papillomavirus vaccination. Am J Prev Med 2014 Dec;47(6):734-744 [FREE Full text] [doi: 10.1016/j.amepre.2014.08.010] [Medline: 25455116]

29. Uthman OA, Sambala EZ, Adamu AA, Ndwandwe D, Wiyeh AB, Olukade T, et al. Does it really matter where you live? A multilevel analysis of factors associated with missed opportunities for vaccination in sub-Saharan Africa. Hum Vaccin Immunother 2018;14(10):2397-2404 [FREE Full text] [doi: 10.1080/21645515.2018.1504524] [Medline: 30059645]

30. Ndwandwe D, Uthman OA, Adamu AA, Sambala EZ, Wiyeh AB, Olukade T, et al. Decomposing the gap in missed opportunities for vaccination between poor and non-poor in sub-Saharan Africa: a multicountry analyses. Hum Vaccin Immunother 2018;14(10):2358-2364 [FREE Full text] [doi: 10.1080/21645515.2018.1467685] [Medline: 29688133]

31. Pancholi J, Birdie R, Guerette J, Chritz S, Sampath V, Crawford J. Landscape analysis of electronic immunization registries. VillageReach. 2020. URL: https://www.comminit.com/ict-4-development/content/

landscape-analysis-electronic-immunization-registries [accessed 2021-12-17]

32. Werner L, Seymour D, Puta C, Gilbert S. Three Waves of Data Use Among Health Workers: The Experience of the Better Immunization Data Initiative in Tanzania and Zambia. Glob Health Sci Pract 2019 Sep;7(3):447-456. [doi:

10.9745/GHSP-D-19-00024] [Medline: $\underline{31558600]}$

33. Pan American Health Organization. Immunization Data: Evidence for Action. A Realist Review of What Works to Improve Data Use for Immunization, Evidence from Low- and Middle Income Countries. Washington, DC: PAHO; 2019.

34. Olorunsaiye CZ, Langhamer MS, Wallace AS, Watkins ML. Missed opportunities and barriers for vaccination: a descriptive analysis of private and public health facilities in four African countries. Pan Afr Med J 2017;27(Suppl 3):6 [FREE Full text] [doi: 10.11604/pamj.supp.2017.27.3.12083] [Medline: 29296141]

35. Ogbuanu IU, Li AJ, Anya BM, Tamadji M, Chirwa G, Chiwaya KW, et al. Can vaccination coverage be improved by reducing missed opportunities for vaccination? Findings from assessments in Chad and Malawi using the new WHO methodology. PLoS One 2019;14(1):e0210648 [FREE Full text] [doi: 10.1371/journal.pone.0210648] [Medline: 30677072]

36. Dang H, Dao S, Carnahan E, Kawakyu N, Duong H, Nguyen T, et al. Determinants of scale-up from a small pilot to a national electronic immunization registry in Vietnam: qualitative evaluation. J Med Internet Res 2020 Sep 22;22(9):e19923 [FREE Full text] [doi: 10.2196/19923] [Medline: $\underline{\text { 32960184] }}$

\author{
Abbreviations \\ BCG: Bacillus Calmette-Guérin \\ BID: Better Immunization Data \\ CQI: continuous quality improvement \\ EIR: electronic immunization registry \\ GADM: Database of Global Administrative Areas \\ LMIC: low- and middle-income country \\ MCV: measles-containing vaccine \\ MOV: missed opportunity for vaccination \\ OPV: oral polio vaccine \\ TImR: Tanzania Immunization Registry \\ WHO: World Health Organization
}

Edited by Y Khader; submitted 28.07.21; peer-reviewed by H Mwanyika, D Ndwandwe, S Dao, N Kawakyu; comments to author 01.10.21; revised version received 15.10.21; accepted 15.10.21; published 21.01.22

Please cite as:

Secor AM, Mtenga H, Richard J, Bulula N, Ferriss E, Rathod M, Ryman TK, Werner L, Carnahan E

Added Value of Electronic Immunization Registries in Low- and Middle-Income Countries: Observational Case Study in Tanzania

JMIR Public Health Surveill 2022;8(1):e32455

URL: https://publichealth.jmir.org/2022/1/e32455

doi: $10.2196 / 32455$

PMID:

(C)Andrew M Secor, Hassan Mtenga, John Richard, Ngwegwe Bulula, Ellen Ferriss, Mansi Rathod, Tove K Ryman, Laurie Werner, Emily Carnahan. Originally published in JMIR Public Health and Surveillance (https://publichealth.jmir.org), 21.01.2022. This 
is an open-access article distributed under the terms of the Creative Commons Attribution License (https://creativecommons.org/licenses/by/4.0/), which permits unrestricted use, distribution, and reproduction in any medium, provided the original work, first published in JMIR Public Health and Surveillance, is properly cited. The complete bibliographic information, a link to the original publication on https://publichealth.jmir.org, as well as this copyright and license information must be included. 